\title{
Using Mathematics to Assess the Impact of Insecticide-based Interventions and Vaccination on Malaria Control
}

\author{
Akash Anickode \\ ${ }^{\dagger}$ School of Mathematical and Statistical Sciences, Arizona State University, Tempe, Arizona, USA.
}

July 21,2020

\begin{abstract}
Malaria, a deadly infectious disease caused by the Plasmodium parasite, remains a major public health challenge in many parts of the world. It causes over 200 million cases and 500,000 fatalities globally each year. There is now a global effort, spearheaded by the World Health Organization and the Bill and Melinda Gates Foundation, to eradicate the disease by 2040. These efforts are primarily based on the large-scale use of insecticide based control measures against the adult female Anopheles mosquito (the malaria vector in humans). This project is based on the use of a basic compartmental model for malaria transmission dynamics to assess various anti-malaria control strategies. The disease-free equilibrium of the model is shown to be locally-asymptotically stable when a certain threshold quantity is less than unity. Furthermore, this equilibrium is globally-asymptotically stable for the special case with no malaria mortality in the human population. Numerical simulations of the model show that the use of a vaccine as a sole anti-malaria strategy may not lead to the elimination of malaria in malaria-endemic setting. However, the study shows that combining vaccination with other vector management strategies (notably insecticide-based mosquito reduction strategies), can lead to such elimination. It is shown that the prospects for the global malaria eradication by 2040 are very promising if the extended vector management strategies (which entails the use of a vaccine together with the insecticide-based mosquito reduction strategies) is implemented at moderate level of effectiveness.
\end{abstract}

\section{Introduction}

Malaria is one of the oldest and deadliest infectious diseases in the world, and continues to impose a major public health and socioeconomic burden in affected areas [43, 52]. The disease is endemic in over 90 countries (in the tropical regions of sub-Saharan Africa, Southeast Asia, and parts of Latin America) [52]. It causes over 200 million cases and 500,000 deaths (mostly among children under the age of five) every year globally [53]. A pproximately $\$ 2.5$ billion is spent annually (globally) on the efforts to control malaria [53]. Due to these major public health and socioeconomic burdens, public health practitioners and organizations, such as the 


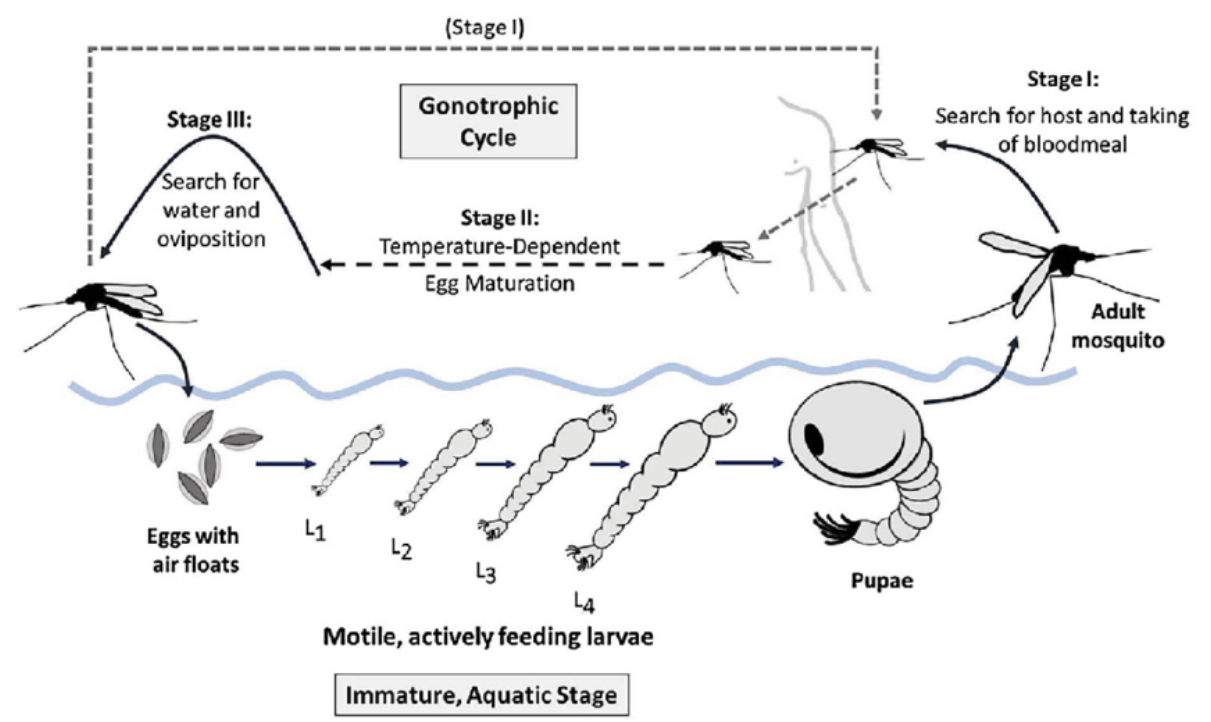

Figure 1: Mosquito Lifecycle [31]

World Health Organization and the Gates Foundation, are working towards the eradication of malaria by the year 2040 [21].

Malaria is a mosquito-borne disease which spreads in human populations when infectious adult female Anopheles mosquitoes bite susceptible humans to obtain protein needed for the development and maturation of eggs [12, 51]. Only about 60 species, out of the over 450 Anopheline species, can successfully serve as a vector for malaria [37]. Tropical Africa faces the most burden of malaria largely because the Anopheline species with high vectorial capacity (e.g. An. gambiae, An. arabiensis, and An. funestus) are very much present there [57]. Malaria is caused by Plasmodium parasites, which infect the blood cells of humans and then replicate [51]. Species of Plasmodium that typically infect humans include $P$. falciparum, $P$. vivax, $P$. ovale, $P$. malariae, and $P$. knowlesi (with $P$. falciparum being the deadliest) [5].

The mosquito lifecycle begins at the immature aquatic stage [9, 31]: Eggs are laid by adult female Anopheles mosquitoes directly onto bodies of water. Then, the eggs hatch into larvae, typically after 2-3 days (2-3 weeks in colder climates). The larvae develop into pupae in about 4-10 days. The pupae undergo metamorphosis and become adult mosquitoes in about 2-4 days. Once the eggs have fully matured into adult mosquitoes, the newly-emerged mosquitoes enter the three-stage gonotrophic cycle [13, 31]. In Stage I of the gonotrophic cycle, the adult female Anopheles mosquito searches for a host to take a blood meal from. In Stage II, the blood meal is digested and the egg develops (at a rate dependent on temperature). Then, in Stage III, the female mosquito searches for an appropriate aquatic environment (breeding habitat), and oviposition (laying of eggs) occurs. Then, the cycle repeats (as depicted in Figure 1 [31]).

Currently, a variety of control strategies are being used to help combat the spread, and mitigate the burden, of malaria. These can be categorized into two main groups, namely preventive and therapeutic [52]. Preventive strategies include the use of insecticide-based control strategies, such as larvicides, adulticides, indoor residual sprays (IRS), long-lasting 
insecticide bednets (LLINs), insect repellent, and anti-malarial prophylaxis [52]. Therapeutic strategies are mainly based on using Artemesinin-based drug therapy to treat humans with clinical symptoms of malaria [52]. The widescale use of the insecticide-based control strategies in malaria-endemic areas has led to significant success against malaria spread and mortality over the last 15 years. Specifically, the widescale use of IRS and LLINs from 20002015 led to a dramatic decrease in the number of malaria cases and malaria mortality [7] (with LLINs accounting for most of the successes). Unfortunately, however, this widespread use of insecticide-based anti-malaria interventions has resulted in Anopheles resistance to all the chemical insecticides used in IRS and LLINs [27, 57]. Other factors that pose important challenges to the global malaria eradication effort include Plasmodium resistance to artemisinin-based drugs [32] and anthropogenic climate change [16]. There are several different strategies to overcome some of these challenges, such as the development of new insecticides for use in IRS and LLINs or the use of biological controls, namely CRISPR-Cas9 gene editing strategy, release of sterile male mosquitoes, or infecting Anopheles mosquitoes with the bacterium Wolbachia [1, 14, 49]. However, there are numerous logistical challenges associated with the large scale deployment of the biological control measures [1, 14, 49]. There is also currently an anti-malarial vaccine being tried in humans (in Kenya, Malawi, and Ghana) called RTS,S [47], albeit its efficacy is expected to be low or average. Consequently, in the absence of a safe and effective vaccine as well as the logistical challenges of deploying genetically-modified mosquitoes into the wild, the global malaria eradication effort is largely focused on the use of insecticide-based interventions [54].

The literature on mathematical modeling of malaria transmission dynamics dates back to the pioneering work of Sir Ronald Ross in the early 1900s [45]. The Ross model, which monitors the temporal dynamics of the total number of infectious humans (denoted by $X(t)$ ) and infectious adult female mosquitoes (denoted by $Z(t)$ ) at time $t$, is given by the following system of nonlinear differential equations:

$$
\begin{aligned}
& \frac{d X}{d t}=\operatorname{mabz}(H-X)-r X, \\
& \frac{d Z}{d t}=\operatorname{acc}(M-Z)-\mu Z,
\end{aligned}
$$

where $m$ is the mosquito per human ratio, $a$ is the biting rate of adult female mosquitoes, $b$ is the probability of infection per bite from an infected mosquito to a susceptible human, $c$ is the probability of infection of a susceptible mosquito per bite on an infected human, $z(t)=Z(t) / M(t)$ is the proportion of infectious mosquitoes at time $t$ (where $M(t)$ is the total number of adult female mosquitoes at time $t$; hence, $M(t)-Z(t)$ is the total number of susceptible mosquitoes at time $t$ ), $H(t)$ is the total number of humans at time $t$ (so that $H(t)-X(t)$ is the total number of susceptible humans), $x(t)=X(t) / H(t)$ is the proportion of infectious humans at time $t, r$ is the human recovery rate and $\mu$ is the natural death rate of mosquitoes. George Macdonald extended the Ross' model in 1957 to include the time lag between the initial infection of the adult female mosquito and the time the mosquito can transmit malaria to humans. That is, Macdonald included the duration of the vector sporogonic cycle [36]. Using the expression for thebasic reproduction number of the model (denoted by $\mathbb{R}_{0}$ ) [33, 34, 35, 36], Ross and Macdonald proposed control strategies 
for effectively controlling the spread of malaria. While Ross emphasized control based on the destruction of breeding habitats for mosquitoes (using larvicides), Macdonald emphasized killing adult mosquitoes before they complete the sporogonic cycle (i.e., Macdonald emphasized the use of adulticides and other strategies, such as the use of bednets, that target the adult mosquito). Numerous other extensions of the Ross-Macdonald modeling framework have been made in the literature to include various other pertinent aspects of the malaria disease, such as drug treatment (and drug resistance) [32], insecticide resistance $[18,27,28,41,50]$, changes in climatic variables [17, 30, 31], mosquito population genetics [27], potential anti-malaria vaccine [4, 29], land-use changes [6], use of biological controls (such as sterile insect technology) against the malaria vector [25], multiple strains of malaria [20], and human mobility [39].

The objective of the current project is to use mathematical modeling approaches to assess the population-level impact of the community-wide implementation of preventive strategies against malaria. In particular, the following strategies will be assessed, implemented singularly or in combinations: larviciding, adulticiding, personal protection against mosquito bites (which includes IRS, LLINs, and insect repellent), and the potential use of an antimalaria vaccine. The aim is to determine whether the concerted global effort to eradicate malaria by 2040 can be achieved using the currently-available preventive resources (i.e., the aforementioned insecticide-based control measures and the potential use of an anti-malaria vaccine). The objective of the project will be achieved through the development, analysis, and simulation of a mathematical model for malaria spread and control in a community. The model to be developed in this project is based on extending the Ross-Macdonald model to incorporate the use of various vector-reduction strategies, such as the use of larvicides, bednets and adulticides, and personal protection against mosquito bites, such as the use of insect repellents and bednets. The paper is organized as follows: The basic model to be used is formulated in Section 2. The basic model is rigorously analyzed, for the asymptotic stability of the associated disease-free equilibrium in Section 3. Numerical simulation results are also reported. The basic model is extended to incorporate an anti-malaria vaccine in Section 4.

\section{Formulation of Basic Model}

The model to be developed in this project is formulated as follows.

\subsection{State Variables of the Model}

The basic model considered in this project is based on subdividing the total human population, denoted by $N_{h}(t)$, into the following mutually-exclusive compartments: susceptible $\left(S_{h}(t)\right)$, exposed $\left(E_{h}(t)\right)$, infectious $\left(I_{h}(t)\right)$, and recovered $\left(R_{h}(t)\right)$ humans, where $t$ represents time. Hence,

$$
N_{h}(t)=S_{h}(t)+E_{h}(t)+I_{h}(t)+R_{h}(t) .
$$

It should be mentioned that "exposed" humans (i.e., those in the $E_{h}(t)$ class) are those that are newly-infected with malaria but have yet to show the clinical symptoms of the disease. 
Furthermore, those in the exposed class are unable to spread the disease, since they have not survived the incubation period yet. That is, they have not yet become infectious. On the other hand, those in the infectious class (i.e., those in the $I_{h}(t)$ class) show the clinical symptoms of malaria and can transmit the disease to susceptible mosquitoes following an effective bite. Similarly, the total mosquito population, denoted by $N_{v}(t)$, is divided into the following mutually-exclusive compartments: susceptible $\left(S_{v}(t)\right)$, exposed $\left(E_{v}(t)\right)$, and infectious $\left(I_{v}(t)\right)$ adult female Anopheles mosquitoes. That is,

$$
N_{v}(t)=S_{v}(t)+E_{v}(t)+I_{v}(t) .
$$

\subsection{Derivation of Equations}

\subsubsection{Rate of change of susceptible human population $S_{h}(t)$}

Humans enter the susceptible population through birth or immigration at a per capita rate $\Pi_{h}$. Humans become infected after an effective bite by an infectious adult female Anopheles mosquito. Let $b$ be the per capita biting rate of the adult female Anopheles mosquitoes and $\beta_{v h}$ be the probability of transmission from an infectious adult female Anopheles mosquito to a susceptible human. Further, let $\mu_{h}$ represents the natural death of humans. Thus, the equation for the rate of change of the susceptible human population is given by the following non-linear differential equation:

$$
\frac{d S_{h}(t)}{d t}=\Pi_{h}-b \beta_{v h} \frac{I_{v}(t)}{N_{h}(t)} S_{h}(t)-\mu_{h} S_{h}(t) .
$$

It is important to mention that the reason the transmission term is normalized by the total host population, $N_{h}(t)$, is because the conservation law of mosquito bites has been imposed. In other words, the conservation law that the total number of bites made by adult female mosquitoes in the community balances the total number of bites received by the human hosts in the community. Further details on the application of this conservation law in mosquitoborne disease models are given in [8, 22].

\subsubsection{Rate of change of exposed human population $E_{h}(t)$}

Humans enter the exposed class after acquiring malaria infection at the rate $b \beta_{v h}$. Exposed humans enter the infectious class after displaying clinical symptoms of malaria at a rate $\sigma_{h}$ (with $\frac{1}{\sigma_{h}}$ representing the average incubation period of the disease). The population of exposed humans is further decreased by natural death (at the rate $\mu_{h}$ ). Thus, the equation for the rate of change of the exposed human population is given by the following non-linear differential equation:

$$
\frac{d E_{h}(t)}{d t}=b \beta_{v h} \frac{I_{v}(t)}{N_{h}(t)} S_{h}(t)-\sigma_{h} E_{h}(t)-\mu_{h} E_{h}(t)
$$

\subsubsection{Rate of change of infectious human population $I_{h}(t)$}

Humans enter the infectious class after developing clinical symptoms of malaria at the rate $\sigma_{h}$. The population of infectious humans is reduced by recovery (at a rate $\gamma_{h}$ ), natural death 
(at the rate $\mu_{h}$ ) and by malaria-induced death (at a rate $\delta_{h}$ ). Thus, the equation for the rate of change of the infectious human population is given by the following linear differential equation:

$$
\frac{d I_{h}(t)}{d t}=\sigma_{h} E_{h}(t)-\gamma_{h} I_{h}(t)-\mu_{h} I_{h}(t)-\delta_{h} I_{h}(t)
$$

\subsubsection{Rate of change of recovered human population $R_{h}(t)$}

The population of recovered humans is generated by the recovery of infectious humans at the rate $\gamma_{h}$, and is reduced by natural death (at the rate $\mu_{h}$ ). Thus, the equation for the rate of change of the recovered human population is given by the following linear differential equation:

$$
\frac{d R_{h}(t)}{d t}=\gamma_{h} I_{h}(t)-\mu_{h} R_{h}(t)
$$

\subsubsection{Rate of change of susceptible mosquito population $S_{v}(t)$}

Susceptible adult female Anopheles mosquitoes are generated through oviposition (following the completion of the immature cycle) at a per capita rate $\Pi_{v}$. The susceptible mosquitoes become infected with malaria, following an effective bite on an infectious human, at a rate $b \beta_{h v}$, where $b$ is the biting rate and $\beta_{h v}$ is the probability of transmission from an infectious human to a susceptible adult female Anopheles mosquito. Furthermore, the population of adult female mosquitoes is diminished by natural death at a rate $\mu_{v}$. Thus, the equation for the rate of change of the susceptible adult female Anopheles mosquito population is given by the following non-linear differential equation:

$$
\frac{d S_{v}(t)}{d t}=\Pi_{v}-b \beta_{h v} \frac{I_{h}(t)}{N_{h}(t)} S_{v}(t)-\mu_{v} S_{v}(t)
$$

\subsubsection{Rate of change of exposed mosquito population $E_{v}(t)$}

Susceptible mosquitoes enter the exposed class, following the acquisition of malaria infection at the rate $b \beta_{v h}$. The population of exposed mosquitoes is decreased by progression to the class of infectious mosquitoes (at a rate $\sigma_{v}$ ) and by natural death (at the rate $\mu_{v}$ ). Thus, the equation for the rate of change of the exposed adult female Anopheles mosquito population is given by the following non-linear differential equation:

$$
\frac{d E_{v}(t)}{d t}=b \beta_{h v} \frac{I_{h}(t)}{N_{h}(t)} S_{v}(t)-\sigma_{v} E_{v}(t)-\mu_{v} E_{v}(t) .
$$

\subsubsection{Rate of change of infectious mosquito population $I_{v}(t)$}

The population of infectious mosquitoes is generated at the rate $\sigma_{v}$ and diminished by natural death (at the rate $\mu_{v}$ ). Thus, the equation for the rate of change of the infectious adult female Anopheles mosquito population is given by the following linear differential equation: 


$$
\frac{d I_{v}(t)}{d t}=\sigma_{v} E_{v}(t)-\mu_{v} I_{v}(t) .
$$

Using the above derivations and assumptions, it follows that the basic model for malaria transmission in a community is given by the following system of non-linear differential equations:

$$
\left\{\begin{array}{l}
\frac{d S_{h}(t)}{d t}=\Pi_{h}-b \beta_{v h} \frac{I_{v}(t)}{N_{h}(t)} S_{h}(t)-\mu_{h} S_{h}(t), \\
\frac{d E_{h}(t)}{d t}=b \beta_{v h} \frac{I_{v}}{N_{h}(t)} S_{h}(t)-\sigma_{h} E_{h}(t)-\mu_{h} E_{h}(t), \\
\frac{d I_{h}(t)}{d t}=\sigma_{h} E_{h}(t)-\gamma_{h} I_{h}(t)-\mu_{h} I_{h}(t)-\delta_{h} I_{h}(t), \\
\frac{d R_{h}(t)}{d t}=\gamma_{h} I_{h}(t)-\mu_{h} R_{h}(t), \\
\frac{d S_{v}(t)}{d t}=\Pi_{v}-b \beta_{h v} \frac{I_{h}(t)}{N_{h}(t)} S_{v}(t)-\mu_{v} S_{v}(t), \\
\frac{d E_{v}(t)}{d t}=b \beta_{h v} \frac{I_{h}(t)}{N_{h}(t)} S_{v}(t)-\sigma_{v} E_{v}(t)-\mu_{v} E_{v}(t), \\
\frac{d I_{v}(t)}{d t}=\sigma_{v} E_{v}(t)-\mu_{v} I_{v}(t) .
\end{array}\right.
$$

A flow diagram of the basic model (2.1) is depicted in Figure 2, and the state variables and parameters of the model are described in Tables 1 and 2, respectively. The main assumptions made in the formulation of the basic model (2.1) include the following:

1. Exponentially-distributed time in all epidemiological compartments.

2. Homogeneous (well-mixed) population. The implication of this assumption is that each mosquito bite has an equal chance of leading to the infection of the susceptible human or in acquiring infection from an infectious human.

3. Malaria recovery induces permanent immunity against future malaria infection. This is a simplifying assumption made for mathematical tractability (since it is known that malaria has a repeated exposure property [3].

4. Exposed humans or vectors do not transmit malaria infection. 


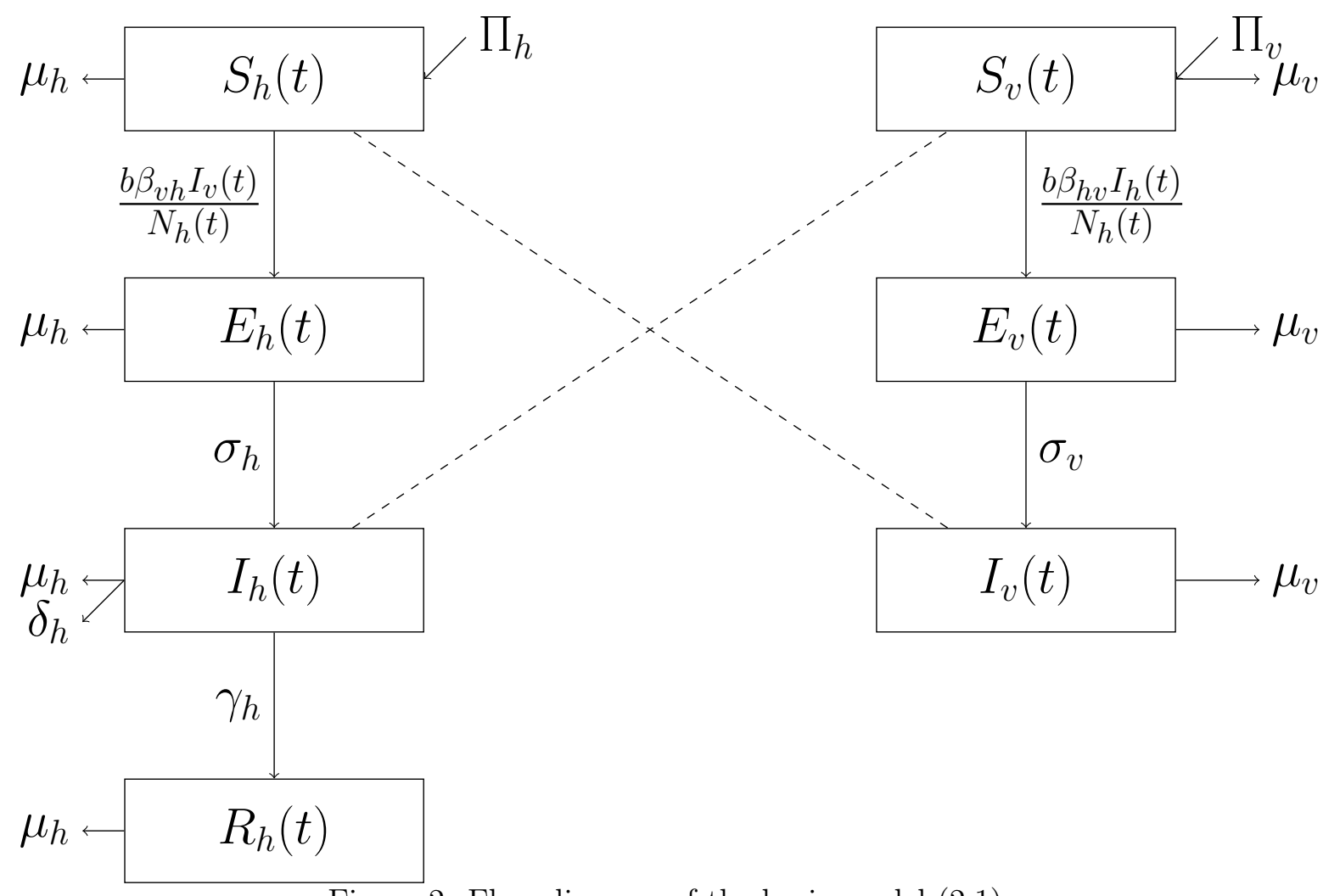

Figure 2: Flow diagram of the basic model (2.1).

\begin{tabular}{|c|l|}
\hline State Variable & Description \\
\hline$S_{h}(t)$ & Population of susceptible humans at time $t$ \\
$E_{h}(t)$ & Population of exposed (newly-infected, but not yet infectious) humans at time $t$ \\
$I_{h}(t)$ & Population of infectious humans at time $t$ \\
$R_{h}(t)$ & Population of recovered humans at time $t$ \\
$S_{v}(t)$ & Population of susceptible adult female Anopheles mosquitoes at time $t$ \\
$E_{v}(t)$ & Population of exposed adult female Anopheles mosquitoes at time $t$ \\
$I_{v}(t)$ & Population of infectious adult female Anopheles mosquitoes at time $t$ \\
\hline
\end{tabular}

Table 1: Description of the state variables of the basic model (2.1). 


\begin{tabular}{|c|c|c|c|}
\hline Parameter & Description & $\begin{array}{l}\text { Baseline } \\
\text { value }\end{array}$ & Reference \\
\hline $\mathrm{b}$ & $\begin{array}{l}\text { Per capita biting rate of } \\
\text { adult female Anopheles } \\
\text { mosquitoes }\end{array}$ & 2.0 per day & {$[11,46]$} \\
\hline$\beta_{h v}\left(\beta_{v h}\right)$ & $\begin{array}{l}\text { Probability of infection per } \\
\text { bite from an infectious hu- } \\
\text { man (mosquito) to a suscep- } \\
\text { tible mosquito (human) }\end{array}$ & $\begin{array}{l}0.48 \quad(0.14) \\
\text { dimension- } \\
\text { less }\end{array}$ & [40] \\
\hline$\Pi_{h}$ & $\begin{array}{l}\text { Recruitment rate of humans } \\
\text { (by birth or immigration) }\end{array}$ & $\begin{array}{l}2.2 \text { humans } \\
\text { per day }\end{array}$ & {$[19,38]$} \\
\hline$\Pi_{v}$ & $\begin{array}{l}\text { Per capita birth rate of } \\
\text { adult female Anopheles } \\
\text { mosquitoes }\end{array}$ & 100 per day & Estimated \\
\hline$\mu_{h}\left(\mu_{v}\right)$ & $\begin{array}{l}\text { Natural death rate for hu- } \\
\text { mans (mosquitoes) }\end{array}$ & $\begin{array}{l}4.405 \times 10^{-5} \\
(1 / 14) \quad \text { per } \\
\text { day }\end{array}$ & {$[10,12]$} \\
\hline$\frac{1}{\sigma_{h}}$ & $\begin{array}{l}\text { Intrinsic incubation period } \\
\text { for humans }\end{array}$ & 14 days & {$[27]$} \\
\hline$\frac{1}{\sigma_{v}}$ & $\begin{array}{l}\text { Extrinsic incubation period } \\
\text { for adult female Anopheles } \\
\text { mosquitoes }\end{array}$ & 10 days & [27] \\
\hline$\gamma_{h}$ & Recovery rate for humans & $\begin{array}{l}0.00658 \text { per } \\
\text { day }\end{array}$ & $\begin{array}{l}{[11,15,26,} \\
31,55,56]\end{array}$ \\
\hline$\delta_{h}$ & $\begin{array}{l}\text { Disease-induced death rate } \\
\text { for humans }\end{array}$ & $\begin{array}{l}9.0 \times 10^{-5} \\
\text { per day }\end{array}$ & [12] \\
\hline
\end{tabular}

Table 2: Description of the parameters of the basic model (2.1). 


\subsection{Basic Qualitative Properties}

In this section, the basic dynamical features of the basic model (2.1) will be explored. Let,

$$
\Omega_{h}=\left\{\left(S_{h}, E_{h}, I_{h}, R_{h}\right) \in \mathbb{R}_{+}^{4}: N_{h} \leq \frac{\Pi_{h}}{\mu_{h}}\right\}
$$

and,

$$
\Omega_{v}=\left\{\left(S_{v}, E_{v}, I_{v}\right) \in \mathbb{R}_{+}^{3}: N_{v} \leq \frac{\Pi_{v}}{\mu_{v}}\right\} .
$$

Further, let $\Omega=\Omega_{h} \cup \Omega_{v}$. We claim the following result.

Theorem 2.1 The closed set $\Omega=\Omega_{h} \cup \Omega_{v}$ is positively-invariant and attracting with respect to the basic model (2.1).

Proof. The proof follows the approach given in [22]. Adding the first four equations and the last three equations of the basic model (2.1) gives, respectively,

$$
\frac{d N_{h}(t)}{d t}=\Pi_{h}-\mu_{h} N_{h}(t)-\delta_{h} I_{h}(t)
$$

and,

$$
\frac{d N_{v}(t)}{d t}=\Pi_{v}-\mu_{v} N_{v}(t)
$$

It can be seen from the above equations that $\frac{d N_{h}(t)}{d t}<0$ if $N_{h}(t)>\frac{\Pi_{h}}{\mu_{h}}$ and $\frac{d N_{v}(t)}{d t}<0$ if $N_{v}(t)>\frac{\Pi_{v}}{\mu_{v}}$. It follows, using a comparison theorem [48], that:

$$
N_{h}(t) \leq N_{h}(0) e^{-\mu_{h}(t)}+\frac{\Pi_{h}}{\mu_{h}}\left(1-e^{-\mu_{h}(t)}\right),
$$

and,

$$
N_{v}(t) \leq N_{v}(0) e^{-\mu_{v}(t)}+\frac{\Pi_{v}}{\mu_{v}}\left(1-e^{-\mu_{v}(t)}\right)
$$

Thus, it follows from the inequalities (2.2) and (2.3) that $N_{h}(t) \leq \frac{\Pi_{h}}{\mu_{h}}$ if $N_{h}(0) \leq \frac{\Pi_{h}}{\mu_{h}}$, and $N_{v}(t) \leq \frac{\Pi_{v}}{\mu_{v}}$ if $N_{v}(0) \leq \frac{\Pi_{v}}{\mu_{v}}$. Thus, the regions $\Omega_{h}$ and $\Omega_{v}$ are positively-invariant with respect to the model (2.1). Hence, the union of these sets, $\Omega=\Omega_{h} \cup \Omega_{v}$, is also positivelyinvariant with respect to the model (2.1). Furthermore, if $N_{h}(t)>\frac{\Pi_{h}}{\mu_{h}}$ and $N_{v}(t)>\frac{\Pi_{v}}{\mu_{v}}$, then the solutions of the model (2.1) enter the regions $\Omega_{h}$ and $\Omega_{v}$ (hence $\Omega$ ) in finite time [22]. Thus, $N_{h}(t)$ approaches $\frac{\Pi_{h}}{\mu_{h}}$ and $N_{v}(t)$ approaches $\frac{\Pi_{v}}{\mu_{v}}$ as $t \rightarrow \infty$. In the former case, the infected variables $\left(E_{h}(t), I_{h}(t), E_{v}(t), I_{v}(t)\right)=(0,0,0,0)$ as $t \rightarrow \infty$. Similarly, in the latter 
case, $\left(E_{v}(t), I_{v}(t)\right)=(0,0)$ as $t \rightarrow \infty$. Thus, the regions $\Omega_{h}$ and $\Omega_{v}$ (hence, $\left.\Omega\right)$ attract all solutions of the basic model (2.1). Hence, the region $\Omega=\Omega_{h} \cup \Omega_{v}$ is attracting with respect to the basic model (2.1). It follows from the above analysis that the basic model (2.1) is well-posed (in $\Omega$ ) both epidemiologically and mathematically. Therefore, it is sufficient to study the dynamics of the basic model (2.1) in the region $\Omega$. Having established the well-posedness of the basic model, the existence and asymptotic stability of its disease-free equilibrium (DFE) will now be analysed.

\section{Analysis of the Basic Model}

\subsection{Local Asymptotic Stability of DFE}

The basic model (2.1) has a DFE, obtained by setting the right-hand sides of the model to zero (with $E_{h}=I_{h}=E_{v}=I_{v}=0$ ), given by

$$
E_{0}=\left(S_{h}^{*}, E_{h}^{*}, I_{h}^{*}, R_{h}^{*}, S_{v}^{*}, E_{v}^{*}, I_{v}^{*}\right)=\left(\frac{\Pi_{h}}{\mu_{h}}, 0,0,0, \frac{\Pi_{v}}{\mu_{v}}, 0,0\right) .
$$

The next generation operator method can be used to analyze the local asymptotic stability of the DFE [42]. Using the notation in [42], the associated matrix $F$ (of new infection terms) and matrix $V$ (of linear transition terms) are given, respectively, by

$$
\mathrm{F}=\left[\begin{array}{cccc}
0 & 0 & 0 & \mathrm{~b} \beta_{v h} \frac{S_{h}^{*}}{N_{h}^{*}} \\
0 & 0 & 0 & 0 \\
0 & \mathrm{~b} \beta_{h v} \frac{S_{v}^{*}}{N_{h}^{*}} & 0 & 0 \\
0 & 0 & 0 & 0
\end{array}\right] \text { and } \mathrm{V}=\left[\begin{array}{cccc}
\sigma_{h}+\mu_{h} & 0 & 0 & 0 \\
-\sigma_{h} & \gamma_{h}+\mu_{h}+\delta_{h} & 0 & 0 \\
0 & 0 & \sigma_{v}+\mu_{v} & 0 \\
0 & 0 & -\sigma_{v} & \mu_{v}
\end{array}\right] .
$$

It then follows that (noting that $S_{h}^{*}=\Pi_{h} / \mu_{h}$ and $S_{v}^{*}=\Pi_{v} / \mu_{v}$ )

$$
\mathbb{R}_{0}=\rho\left(F V^{-1}\right)=\sqrt{\mathbb{R}_{0, h v} \mathbb{R}_{0, v h}},
$$

where,

$$
\mathbb{R}_{0, h v}=\left(\frac{b \beta_{h v} \Pi_{v} \mu_{h}}{\Pi_{h} \mu_{v}}\right)\left(\frac{\sigma_{h}}{\sigma_{h}+\mu_{h}}\right)\left(\frac{1}{\gamma_{h}+\delta_{h}+\mu_{h}}\right)
$$

and,

$$
\mathbb{R}_{0, v h}=\left(b \beta_{v h}\right)\left(\frac{\sigma_{v}}{\sigma_{v}+\mu_{v}}\right)\left(\frac{1}{\mu_{v}}\right) .
$$

The result below follows from Theorem 2 of [42].

Theorem 3.1 The DFE of the basic model (2.1), denoted by $E_{0}$, is locally-asymptotically stable if $\mathbb{R}_{0}<1$, and unstable if $\mathbb{R}_{0}>1$. 
The quantity $\mathbb{R}_{0}$ is referred to as the basic reproduction number of the model (2.1) [2]. It represents the average number of new malaria cases generated by an infectious human (or mosquito) when introduced into a completely susceptible mosquito (or human) population. The reproduction number of the basic model $(2.1)\left(\mathbb{R}_{0}\right)$ is the geometric mean of the reproduction numbers for human-to-vector $\left(\mathbb{R}_{0, h v}\right)$ and vector-to-human $\left(\mathbb{R}_{0, v h}\right)$ interactions. The geometric mean arises from the fact that two generations are required to complete the human-vector-human or vector-human-vector malaria transmission cycle.

\subsection{Interpretation of the reproduction number $\left(\mathbb{R}_{0}\right)$}

The terms in $\mathbb{R}_{0, h v}$ and $\mathbb{R}_{0, v h}$ are interpreted epidemiologically as follows. The first term in $\mathbb{R}_{0, h v}$ represents the infection rate from an infectious human to a susceptible vector. The second term represents the probability that an individual in the exposed class $\left(E_{h}(t)\right)$ survives long enough to enter the infectious class $\left(I_{h}(t)\right)$. Finally, the third term represents the average duration of an individual in the infectious class $\left(I_{h}(t)\right)$. The product of these three terms gives $\mathbb{R}_{0, h v}$.

Similarly, the first term in $\mathbb{R}_{0, v h}$ represents the infection rate from an infectious vector to a susceptible human. The second term represents the probability that a vector in the exposed class $\left(E_{h}(t)\right)$ survives long enough to enter the infectious class $\left(I_{h}(t)\right)$. Finally, the third term represents the average duration of a vector in the infectious class $\left(I_{h}(t)\right)$. The product of these three terms gives $\mathbb{R}_{0, v h}$.

The epidemiological implication of Theorem 3.1 is that a small influx of infected individuals (i.e. in the basin of attraction of the disease-free equilibrium, $E_{0}$ ) will not generate a large outbreak in the community when the threshold quantity is less than unity. In order for the effective control of the disease to be independent of the initial sizes of the sub-populations of the basic model (2.1), a global asymptotic stability result has to be established for the disease-free equilibrium $\left(E_{0}\right)$. This is done below.

\subsection{Global Asymptotic Stability of DFE}

It is convenient to define $\widetilde{\mathbb{R}}_{0}=\left.\mathbb{R}_{0}\right|_{\delta_{h}=0}$. We claim the following result:

Theorem 3.2 The DFE of the basic model 2.1, $E_{0}$, is globally-asymptotically stable in $\Omega$ whenever $\widetilde{\mathbb{R}}_{0}<1$.

Proof. The proof of Theorem 3.2 is based on using the approach in [24]. Consider the basic model (2.1) with $\delta_{h}=0$. Thus, $\frac{d N_{h}(t)}{d t}=\Pi_{h}-\mu_{h} N_{h}$. Hence,

$$
\limsup _{t \rightarrow \infty} N_{h}(t)=N_{h}^{*}=\frac{\Pi_{h}}{\mu_{h}} .
$$

From now on, $N_{h}(t)$ will be replaced by its limiting value $N_{h}^{*}$. Further, let $\widetilde{\mathbb{R}}_{0}<1$. It is convenient to rewrite the equations for the rate of change of the infected populations of the 
basic model (2.1) as follows:

$$
\frac{d}{d t}\left(\begin{array}{c}
E_{h}(t) \\
I_{h}(t) \\
E_{v}(t) \\
I_{v}(t)
\end{array}\right)=\left(\begin{array}{c}
(F-V) E_{h}(t) \\
I_{h}(t) \\
E_{v}(t) \\
I_{v}(t)
\end{array}\right)-M\left(\begin{array}{c}
E_{h}(t) \\
I_{h}(t) \\
E_{v}(t) \\
I_{v}(t)
\end{array}\right),
$$

where the next generation matrices $F$ and $V$ are as defined in Section 3.1 and the nonnegative matrix, $M$, is given by:

$$
M=\left[\begin{array}{cccc}
0 & 0 & 0 & b \beta_{v h}\left(1-\frac{S_{h}(t)}{N_{h}(t)}\right) \\
0 & 0 & 0 & 0 \\
0 & \frac{b \beta_{h v}}{N_{h}^{*}}\left(S_{v}^{*}-S_{v}(t)\right) & 0 & 0 \\
0 & 0 & 0 & 0
\end{array}\right] .
$$

Since $S_{v}(t) \leq S_{v}^{*}$ and $S_{h}(t) \leq N_{h}(t)$ for all $t>0$ in $\Omega$, it follows that the matrix $\mathrm{M}$ is non-negative. Hence, Equation (3.2) can be rewritten as:

$$
\frac{d}{d t}\left(\begin{array}{c}
E_{h}(t) \\
I_{h}(t) \\
E_{v}(t) \\
I_{v}(t)
\end{array}\right) \leq(F-V)\left(\begin{array}{c}
E_{h}(t) \\
I_{h}(t) \\
E_{v}(t) \\
I_{v}(t)
\end{array}\right) .
$$

Now, consider the linear ODE system given by Equation (3.3), with the inequality replaced by equality. If $\widetilde{\mathbb{R}}_{0}<1$, then $\rho\left(F V^{-1}\right)<1$, which implies that all of the eigenvalues of the matrix $F-V$ are negative. From this, it follows that the system given by Equation (3.3) is stable whenever $\widetilde{\mathbb{R}}_{0}<1$. Thus, $\left(E_{h}(t), I_{h}(t), E_{v}(t), I_{v}(t)\right) \rightarrow(0,0,0,0)$ as $t \rightarrow \infty$. Applying a standard comparison theorem [48], we see that $\left(E_{h}(t), I_{h}(t), E_{v}(t), I_{v}(t)\right) \rightarrow(0,0,0,0)$ for the nonlinear system given by the equations for $\frac{d E_{h}(t)}{d t}, \frac{d I_{h}(t)}{d t}, \frac{d E_{v}(t)}{d t}$, and $\frac{d I_{v}(t)}{d t}$ in the basic model (2.1). By substituting $E_{h}(t)=I_{h}(t)=E_{v}(t)=I_{v}(t)=0$ into the equations for $S_{h}(t)$ and $S_{v}(t)$ in the basic model (2.1), it can be seen that $S_{h}(t) \rightarrow \frac{\Pi_{h}}{\mu_{h}}$ and $S_{v}(t) \rightarrow \frac{\Pi_{v}}{\mu_{v}}$ as $t \rightarrow \infty$. Thus, the DFE of the basic model (2.1), $E_{0}$, is globally asymptotically stable in $\Omega$ whenever $\widetilde{\mathbb{R}}_{0}<1$.

The epidemiological implication of Theorem 3.2 is that, for the special case of the basic model (2.1) with $\delta_{h}=0$, bringing (and maintaining) the reproduction threshold $\mathbb{R}_{0}$ to a value less than unity is necessary and sufficient for the effective control (or elimination) of the disease. It should be mentioned that the result in Theorem 3.2 may not hold if the assumption $\delta_{h}=0$ is relaxed. This is because mosquito-borne disease models, such as the basic model (2.1), undergo the phenomenon of backward bifurcation if the disease-induced mortality in the host population $\left(\delta_{h}\right.$ in the case of the basic model $(2.1)$ ) is not set to zero or is not negligible $[22,23]$. In a backward bifurcation situation, disease control when the reproduction number of the model is less than unity depends on the initial sizes of the subpopulations of the model. Thus, the analysis in Theorem 3.2 shows that $\delta_{h}>0$ is necessary for the presence of backward bifurcation in the basic model (2.1). 
It can be seen from the expression for $\mathbb{R}_{0}$ in Equation (3.1) that the value of $\widetilde{\mathbb{R}}_{0}=\left.\mathbb{R}_{0}\right|_{\delta_{h}=0}$ can be reduced by reducing the biting rate $(b)$, the probability of transmission per bite on a susceptible human $\left(\beta_{h v}\right)$, the probability of infection per bite on an infectious human $\left(\beta_{v h}\right)$, and mosquito birth rate $\left(\Pi_{v}\right)$. It can also be reduced by increasing the recovery rate $\left(\gamma_{h}\right)$ and the death rate of mosquitoes $\left(\mu_{v}\right)$. Hence, control strategies, such as the use of larvicides (reduces $\Pi_{v}$ ), adulticides (increases $\mu_{v}$ ), long-lasting insecticidal nets (decreases $b$ ), insect repellents (decreases $b$ ), vaccination (reduces $\beta_{h v}$ and $\beta_{v h}$ ), and treatment (increases $\gamma_{h}$ ) can

lead to effective control of the disease provided they bring (and maintain) $\widetilde{\mathbb{R}}_{0}$ to a value less than unity. The result in Theorem 3.2 is numerically-illustrated in Figure 3, where numerous initial conditions of the special case of the basic model $(2.1)$, with $\delta_{h}=0$, are shown to converge to the DFE, $E_{0}$ (when the parameters were chosen such that $\widetilde{\mathbb{R}}_{0}<1$ ).

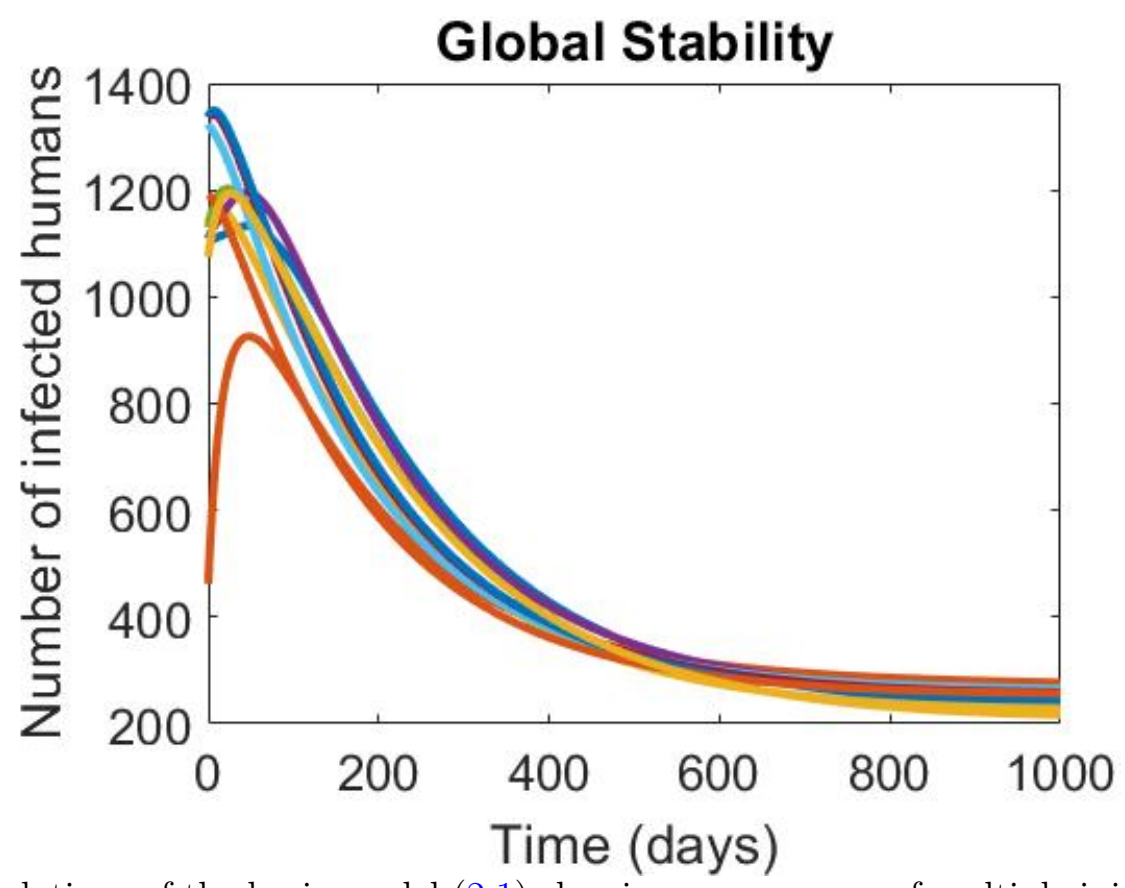

Figure 3: Simulations of the basic model (2.1) showing convergence of multiple initial conditions to the DFE, $E_{0}$. Parameter values used are as given by the baseline values in Table 2 , with $\delta_{h}=0$ and $b=0.5$ (so that, $\widetilde{\mathbb{R}}_{0}=0.762<1$ ).

\subsection{Numerical Simulations of the Basic Model}

In this section, the basic model (2.1) will be simulated using the baseline values in Table 2 (unless otherwise stated) to assess the population-level impact of various anti-malaria control strategies. In particular, the following vector-based control strategies will be assessed: larvicides, adulticides, personal protection against mosquito bites, and an integrated vector management strategy (IVM). First of all, simulating the basic model (2.1), using the baseline values of the parameters in Table 2 , shows convergence of the initial solutions to an endemic equilibrium (where the number of infected humans at steady-state is non-zero), as depicted in Figure 4. For this simulation, the value of the reproduction number of the model is 
$\mathbb{R}_{0}=3.047>1$. It is intuitive that the disease will persist in the population if $\mathbb{R}_{0}>1$, since, in this case, each infected individual will generate, on average, three other new infections in humans (via the vector) or three infections in the vector.

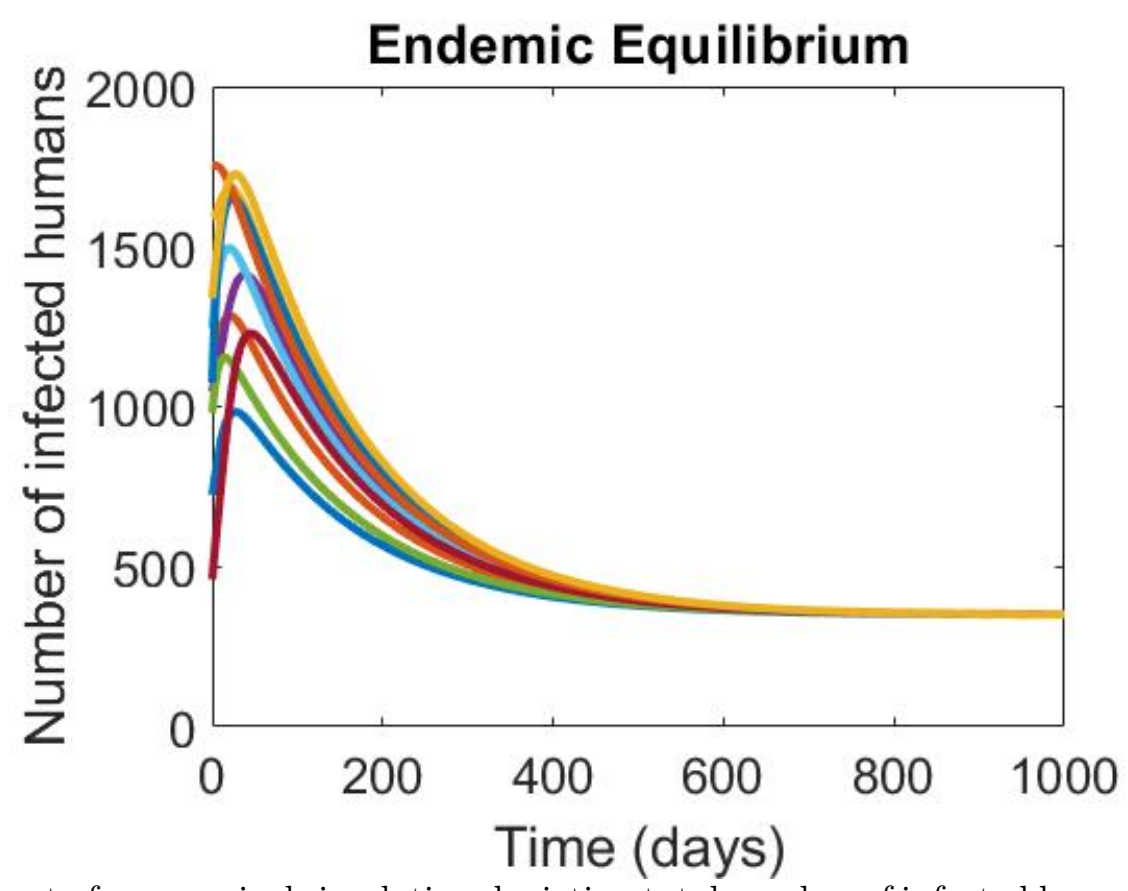

Figure 4: Output of a numerical simulation depicting total number of infected humans $\left(E_{h}(t)+I_{h}(t)\right)$ as a function of time for multiple initial conditions. Parameter values are as given by the baseline values in Table 2 (so that $\mathbb{R}_{0}=3.047$ ).

\subsubsection{Effect of larviciding}

Larviciding is a control strategy that targets mosquito larvae, killing them before they can mature into pupae (which, later, metamorphose into adult mosquitoes). Therefore, in the context of the basic model (2.1), larviciding reduces the per capita birth rate of mosquitoes $\left(\Pi_{v}\right)$. Consequently, larviciding is incorporated into the basic model (2.1) by rescaling the parameter $\Pi_{v}$ as follows:

$$
\Pi_{v} \rightarrow \Pi_{v}\left(1-e_{l} c_{l}\right)
$$

where $0 \leq e_{l} \leq 1$ represents the efficacy of the larvicides and $0 \leq c_{l} \leq 1$ represents the coverage of the larvicides in the community. Using Equation (3.4) in the basic model (2.1) shows that the reproduction number $\mathbb{R}_{0}$ now becomes:

$$
\mathbb{R}_{0, l}=\sqrt{\left(R_{0, h v, l}\right)\left(R_{0, v h, l}\right)},
$$

where,

$$
\mathbb{R}_{0, h v, l}=\left(\frac{b \beta_{h v} \Pi_{v}\left(1-e_{l} c_{l}\right) \mu_{h}}{\Pi_{h} \mu_{v}}\right)\left(\frac{\sigma_{h}}{\sigma_{h}+\mu_{h}}\right)\left(\frac{1}{\gamma_{h}+\delta_{h}+\mu_{h}}\right)
$$




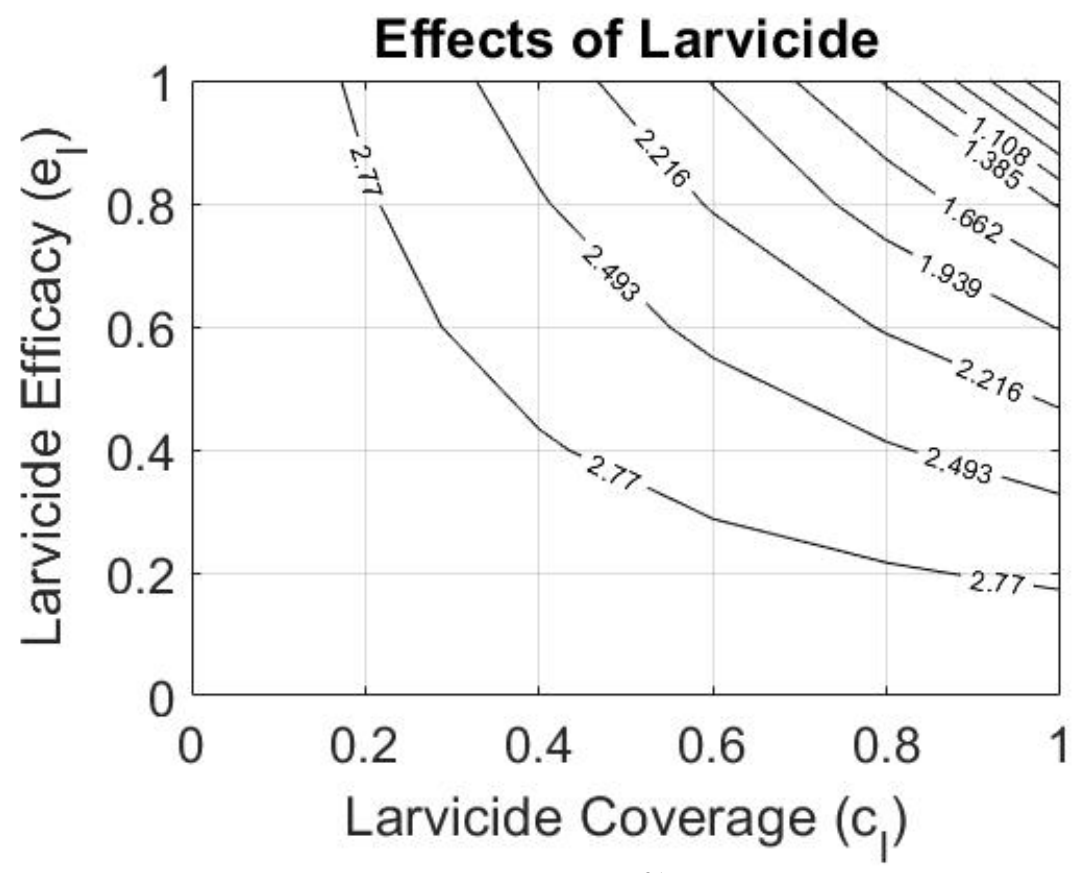

Figure 5: Contour plot of the reproduction number $\widetilde{\mathbb{R}}_{0, l}$ as a function of larvicide efficacy $\left(e_{l}\right)$ and coverage $\left(c_{l}\right)$. Other parameter values used are as given by the baseline values in Table 2, with $\delta_{h}=0$.

and,

$$
\mathbb{R}_{0, v h, l}=\left(b \beta_{v h}\right)\left(\frac{\sigma_{v}}{\sigma_{v}+\mu_{v}}\right)\left(\frac{1}{\mu_{v}}\right) .
$$

It should be noted that when $e_{l}=c_{l}=0$, the quantity $\mathbb{R}_{0, l}$ reduces to $\mathbb{R}_{0}$. Let $\widetilde{\mathbb{R}}_{0, l}=\left.\mathbb{R}_{0, l}\right|_{\delta_{h}=0}$. Figure 5 depicts a contour plot of $\widetilde{\mathbb{R}}_{0, l}$ as a function of larvicide efficacy $\left(e_{l}\right)$ and coverage $\left(c_{l}\right)$. This figure shows that $\widetilde{\mathbb{R}}_{0, l}$ decreases with increasing larvicide efficacy and coverage. Further, this figure shows that very high coverage and efficacy of larvicides will be needed to achieve effective control of the disease in the community (i.e. bring and maintain $\widetilde{\mathbb{R}}_{0, l}<1$ ). Hence, the use of larvicides alone seems to be realistically infeasible to singularly lead to the effective control of the disease in the community.

\subsubsection{Effect of adulticiding}

Adulticiding is a control strategy that targets the killing of adult mosquitoes. Therefore, in the context of the basic model (2.1), adulticiding increases the natural death rate of adult mosquitoes $\left(\mu_{v}\right)$. Consequently, adulticiding is incorporated into the basic model (2.1) by rescaling the parameter $\mu_{v}$ as follows:

$$
\mu_{v} \rightarrow \mu_{v}\left(1+e_{a} c_{a}\right)
$$

where $0 \leq e_{a} \leq 1$ represents the efficacy of the adulticides and $0 \leq c_{a} \leq 1$ represents the 


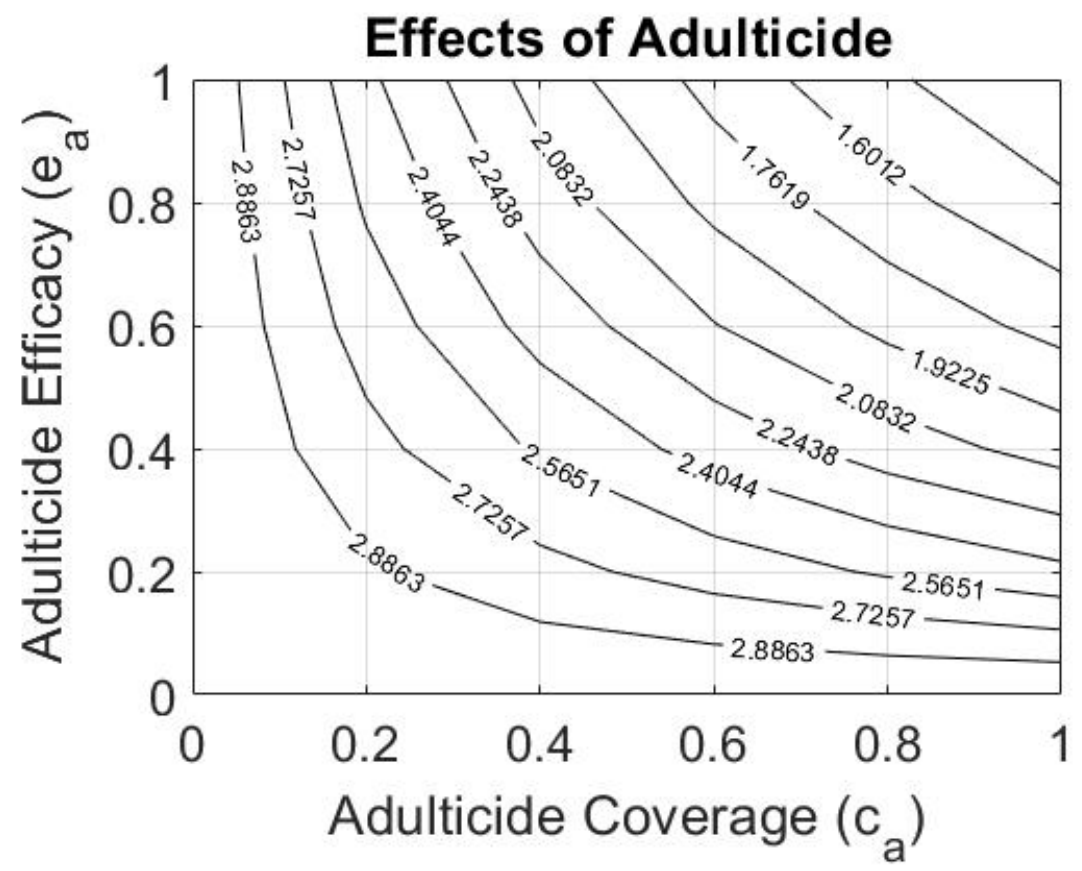

Figure 6: Contour plot of the reproduction number $\widetilde{\mathbb{R}}_{0, a}$ as a function of adulticide efficacy $\left(e_{l}\right)$ and coverage $\left(c_{l}\right)$. Other parameter values used are as given by the baseline values in Table 2, with $\delta_{h}=0$.

coverage of the adulticides in the community. Using Equation (3.5) in the basic model (2.1) shows that the reproduction number $\mathbb{R}_{0}$ now becomes:

$$
\mathbb{R}_{0, a}=\sqrt{\left(R_{0, h v, a}\right)\left(R_{0, v h, a}\right)}
$$

where,

$$
\mathbb{R}_{0, h v, a}=\left(\frac{b \beta_{h v} \Pi_{v} \mu_{h}}{\Pi_{h} \mu_{v}\left(1+e_{a} c_{a}\right)}\right)\left(\frac{\sigma_{h}}{\sigma_{h}+\mu_{h}}\right)\left(\frac{1}{\gamma_{h}+\delta_{h}+\mu_{h}}\right)
$$

and,

$$
\mathbb{R}_{0, v h, a}=\left(b \beta_{v h}\right)\left(\frac{\sigma_{v}}{\sigma_{v}+\mu_{v}\left(1+e_{a} c_{a}\right)}\right)\left(\frac{1}{\mu_{v}\left(1+e_{a} c_{a}\right)}\right)
$$

It should be noted that when $e_{a}=c_{a}=0$, the quantity $\mathbb{R}_{0, a}$ reduces to $\mathbb{R}_{0}$. Let $\widetilde{\mathbb{R}}_{0, a}=$ $\left.\mathbb{R}_{0, a}\right|_{\delta_{h}=0}$. Figure 6 depicts a contour plot of $\widetilde{\mathbb{R}}_{0, a}$ as a function of adulticide efficacy $\left(e_{a}\right)$ and coverage $\left(c_{a}\right)$. This figure shows that $\widetilde{\mathbb{R}}_{0, a} \mathrm{~d}$ ecreases w ith increasing a dulticide efficacy and coverage. Further, this figure s hows t hat, e ven w ith v ery h igh c overage and efficacy, adulticiding alone is unable to achieve effective control of the disease in the community (i.e. bring and maintain $\widetilde{\mathbb{R}}_{0, a}<1$ ). Hence, the use of adulticides alone is infeasible to effectively control the disease. 


\subsubsection{Effect of personal protection against mosquito bite}

Personal protection involves the application of insect repellent on humans to repel adult female mosquitoes from biting the humans. The use of personal protection aims to decrease the biting rate $(b)$ of mosquitoes. In the context of the basic model (2.1), the use of personal protection is incorporated by re-scaling the parameter $b$ as follows:

$$
b \rightarrow b\left(1-e_{p} c_{p}\right)
$$

where $0 \leq e_{p} \leq 1$ represents the efficacy of the personal protection and $0 \leq c_{p} \leq 1$ represents the coverage of the personal protection in the community. Using Equation (3.6) in the basic model (2.1) shows that the reproduction number now becomes:

$$
\mathbb{R}_{0, p}=\sqrt{\left(R_{0, h v, p}\right)\left(R_{0, v h, p}\right)}
$$

where,

$$
\mathbb{R}_{0, h v, p}=\left(\frac{b\left(1-e_{p} c_{p}\right) \beta_{h v} \Pi_{v} \mu_{h}}{\Pi_{h} \mu_{v}}\right)\left(\frac{\sigma_{h}}{\sigma_{h}+\mu_{h}}\right)\left(\frac{1}{\gamma_{h}+\delta_{h}+\mu_{h}}\right)
$$

and,

$$
\mathbb{R}_{0, v h, p}=\left(b\left(1-e_{p} c_{p}\right) \beta_{v h}\right)\left(\frac{\sigma_{v}}{\sigma_{v}+\mu_{v}}\right)\left(\frac{1}{\mu_{v}}\right) .
$$

It should be noted that when $e_{p}=c_{p}=0$, the quantity $\mathbb{R}_{0, p}$ reduces to $\mathbb{R}_{0}$. Let $\widetilde{\mathbb{R}}_{0, p}=$ $\left.\mathbb{R}_{0, p}\right|_{\delta_{h}=0}$. Figure 7 depicts a contour plot of $\widetilde{\mathbb{R}}_{0, p}$ as a function of personal protection efficacy $\left(e_{p}\right)$ and coverage $\left(c_{p}\right)$. This figure shows that $\widetilde{\mathbb{R}}_{0, p}$ decreases with increasing personal protection efficacy and coverage. Further, this figure shows that very high coverage and efficacy of personal protection will be needed to achieve effective control of the disease in the community (i.e. bring and maintain $\widetilde{\mathbb{R}}_{0, p}<1$ ). Hence, the use of personal protection alone seems to be realistically infeasible to singularly lead to the effective control of the disease in the community.

\subsubsection{Effect of integrated vector management strategy (IVM)}

Although the singular use of any of the vector-based strategies discussed earlier proved incapable of leading to effective control (or elimination) of malaria in a community, the use of a combination of these strategies may produce the desired result. In this section, we simulate the combined use of larvicides, adulticides, and personal protection against mosquito bites. The following three effectiveness levels of IVM are considered:

- Low Effectiveness Level: Larvicides at 100\% efficacy and 25\% coverage (i.e. $e_{l}=1$ and $c_{l}=0.25$ ) and adulticides at $50 \%$ efficacy and $25 \%$ coverage (i.e. $e_{a}=0.5$ and $\left.c_{a}=0.25\right)$.

- Moderate Effectiveness Level: Larvicides at $100 \%$ efficacy and $50 \%$ coverage (i.e. $e_{l}=1$ and $\left.c_{l}=0.5\right)$ and adulticides at $50 \%$ efficacy and $50 \%$ coverage (i.e. $e_{a}=0.5$ and $\left.c_{a}=0.5\right)$. 


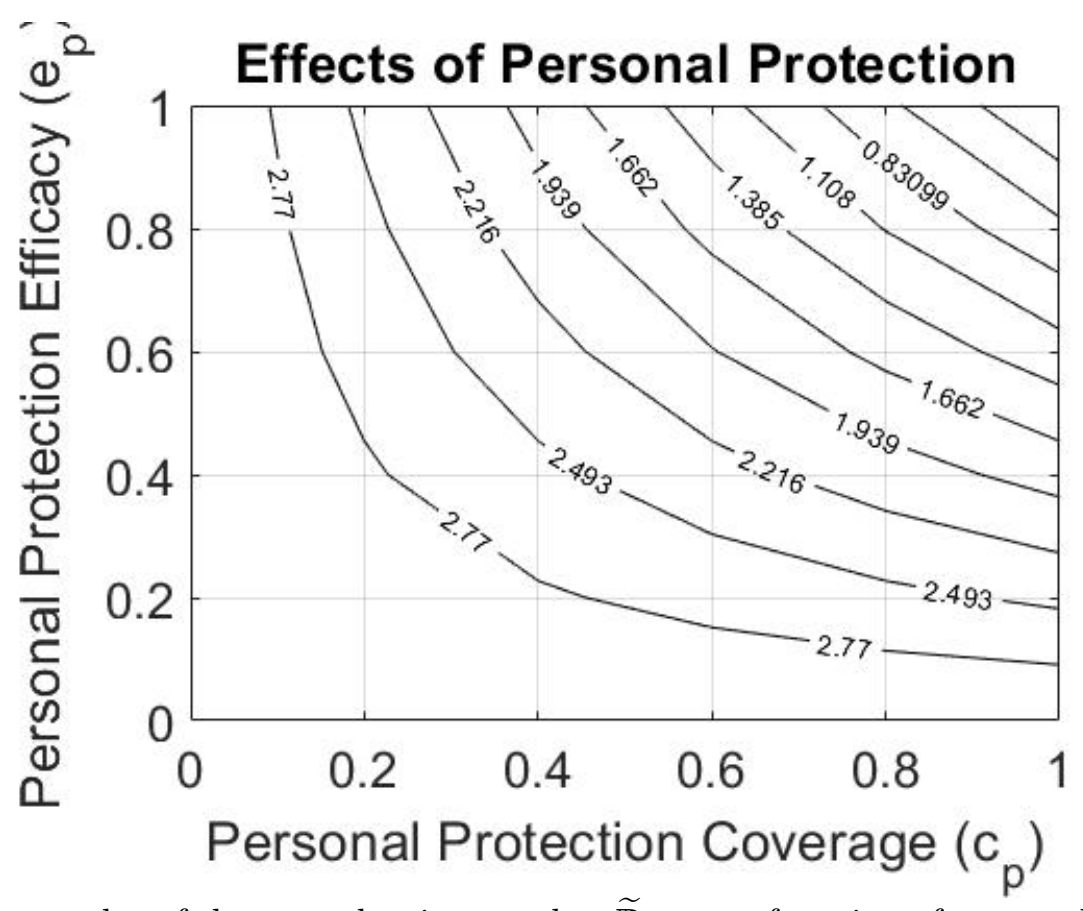

Figure 7: Contour plot of the reproduction number $\widetilde{\mathbb{R}}_{0, p}$ as a function of personal protection efficacy $\left(e_{p}\right)$ and coverage $\left(c_{p}\right)$. Other parameter values used are as given by the baseline values in Table 2 , with $\delta_{h}=0$.

- High Effectiveness Level: Larvicides at $100 \%$ efficacy and $75 \%$ coverage (i.e. $e_{l}=1$ and $\left.c_{l}=0.75\right)$ and adulticides at $50 \%$ efficacy and $75 \%$ coverage (i.e. $e_{a}=0.5$ and $\left.c_{a}=0.75\right)$.

For each of the above IVM effectiveness levels, various values for personal protection efficacy $\left(e_{p}\right)$ and coverage $\left(c_{p}\right)$ will be used. Applying the modifications to the basic model (2.1) to include the implementation of control strategies based on the use of larivicides, adulticides, and personal protection against mosquito bites, it follows that the reproduction number for the IVM strategy, denoted $\mathbb{R}_{0, m}$, is given by:

$$
\mathbb{R}_{0, m}=\sqrt{\left(R_{0, h v, m}\right)\left(R_{0, v h, m}\right)}
$$

where,

$$
\mathbb{R}_{0, h v, m}=\left(\frac{b\left(1-e_{p} c_{p}\right) \beta_{h v} \Pi_{v}\left(1-e_{l} c_{l}\right) \mu_{h}}{\Pi_{h} \mu_{v}\left(1+e_{a} c_{a}\right)}\right)\left(\frac{\sigma_{h}}{\sigma_{h}+\mu_{h}}\right)\left(\frac{1}{\gamma_{h}+\delta_{h}+\mu_{h}}\right),
$$

and,

$$
\mathbb{R}_{0, v h, m}=\left(b\left(1-e_{p} c_{p}\right) \beta_{v h}\right)\left(\frac{\sigma_{v}}{\sigma_{v}+\mu_{v}\left(1+e_{a} c_{a}\right)}\right)\left(\frac{1}{\mu_{v}\left(1+e_{a} c_{a}\right)}\right) .
$$

It is convenient to define $\widetilde{\mathbb{R}}_{0, m}=\left.\mathbb{R}_{0, m}\right|_{\delta_{h}=0}$.

Figure 8a depicts a contour plot of $\widetilde{\mathbb{R}}_{0, m}$ as a function of personal protection efficacy $\left(e_{p}\right)$ and coverage $\left(c_{p}\right)$ for the low effectiveness level of the IVM strategy. The figure shows that 


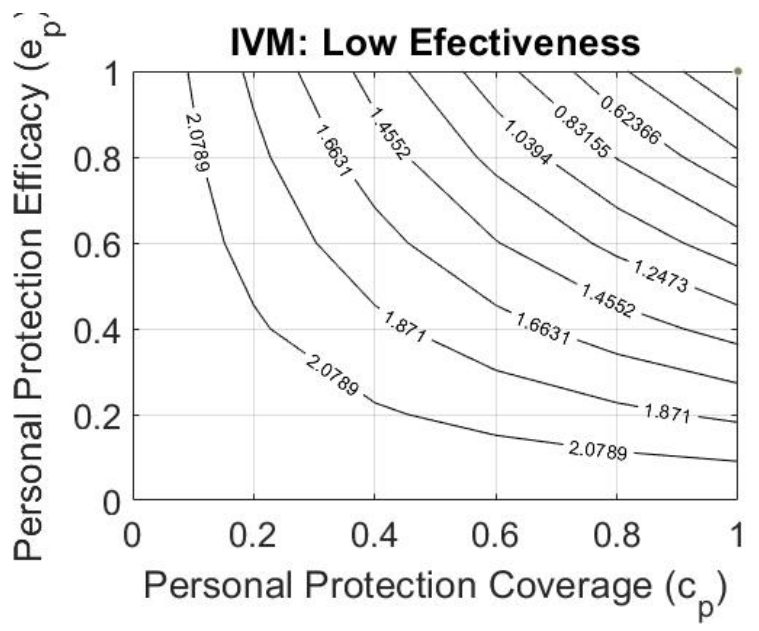

(a)

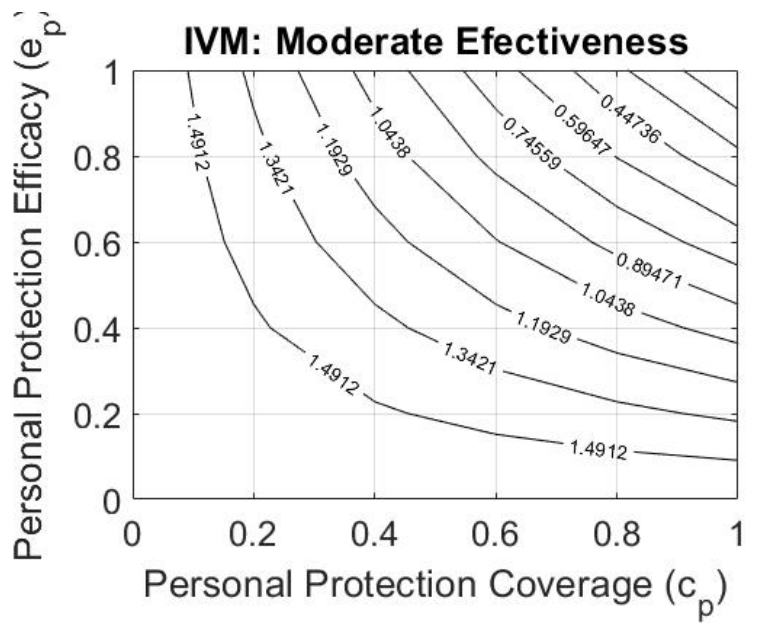

(b)

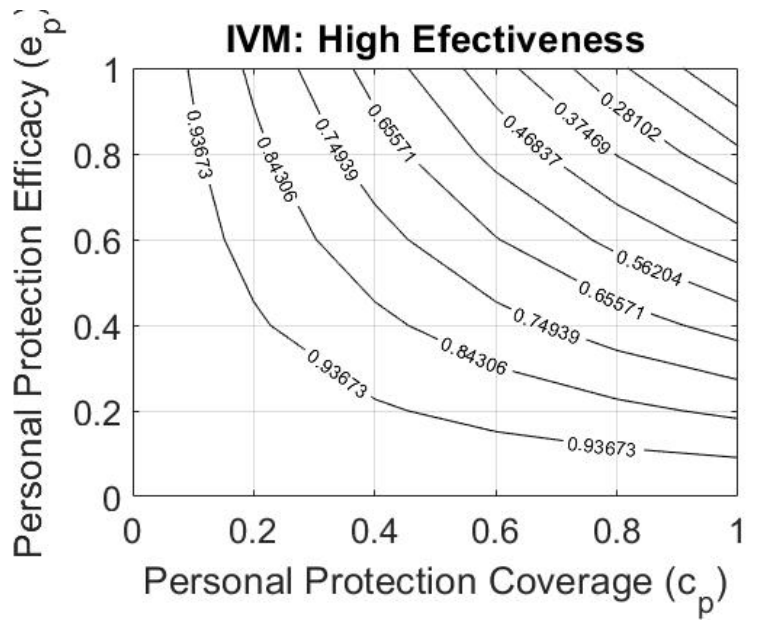

(c)

Figure 8: Simulations of the basic model (2.1) for the impact of IVM. Contour plot of the reproduction number $\left(\widetilde{\mathbb{R}}_{0, m}\right)$ as a function of personal protection efficacy $\left(e_{p}\right)$ and coverage $\left(c_{p}\right)$. Other parameter values are as given by the baseline values in Table 2 , with $\delta_{h}=0$ and various values of $e_{p}$ and $c_{p}$. (a) Low effectiveness level of IVM. (b) Moderate effectiveness level of IVM. (c) High effectiveness level of IVM. 
a high efficacy and coverage of personal protection is necessary to achieve effective control of the disease in a community (i.e. bring and maintain $\widetilde{\mathbb{R}}_{0, m}<1$ ). In particular, if personal protection efficacy is fixed at $80 \%$, a personal protection coverage greater than $70.4 \%$ will be needed. Hence, the IVM strategy at the low effectiveness level may not lead to effective control of the disease.

Figure $8 \mathrm{~b}$ depicts a contour plot of $\widetilde{\mathbb{R}}_{0, m}$ as a function of personal protection efficacy $\left(e_{p}\right)$ and coverage $\left(c_{p}\right)$ for the moderate effectiveness level of the IVM strategy. The figure shows that a moderately high efficacy and coverage of personal protection is necessary to achieve effective control of the disease in a community (i.e. bring and maintain $\widetilde{\mathbb{R}}_{0, m}<1$ ). In particular, if personal protection efficacy is fixed at $80 \%$, a personal protection coverage greater than $48.8 \%$ will be needed. Hence, the IVM strategy at the moderate effectiveness level can potentially lead to effective control of the disease.

Figure 8c depicts a contour plot of $\widetilde{\mathbb{R}}_{0, m}$ as a function of personal protection efficacy $\left(e_{p}\right)$ and coverage $\left(c_{p}\right)$ for the high effectiveness level of the IVM strategy. The figure shows that a very low efficacy and coverage of personal protection is necessary to achieve effective control of the disease in a community (i.e. bring and maintain $\widetilde{\mathbb{R}}_{0, m}<1$ ). In particular, if personal protection efficacy is fixed at $80 \%$, a personal protection coverage greater than $3.7 \%$ will be needed. Hence, the IVM strategy at the high effectiveness level is very likely to lead to effective control of the disease.

\section{Vaccination Model (Extension of Basic Model)}

Although there is currently no safe and effective vaccine being widely used in humans against malaria, a candidate vaccine known as RTS,S is undergoing clinical trials in humans in Kenya, Malawi, and Ghana [47]. This fact motivates the need to extend the basic model (2.1) to incorporate a possible anti-malaria vaccine.

\subsection{Formulation of Vaccination Model}

The basic model (2.1) is now extended to include a compartment, $V_{h}(t)$, for individuals vaccinated against malaria at time $t$. The population of vaccinated individuals is generated by the vaccination of susceptible individuals at a rate $\eta_{h}$. It is decreased by breakthrough infection (where a vaccinated susceptible individual becomes infected) at a rate $\left(1-\epsilon_{h}\right) b \beta_{v h}$, where $b$ and $\beta_{h v}$ are as defined in Section 2.2 and $0<\epsilon_{h} \leq 1$ is the vaccine protective efficacy against the acquisition of malaria infection. Further, the population is decreased due to the waning of the vaccine (at a rate $\omega_{h}$ ) and by natural death (at the rate $\mu_{h}$ ). Thus, the equation for the rate of change of the population of vaccinated susceptible individuals $\left(V_{h}(t)\right)$ is given by the non-linear differential equation:

$$
\frac{d V_{h}(t)}{d t}=\eta_{h} S_{h}(t)-\left(1-\epsilon_{h}\right) b \beta_{h v} \frac{I_{v}(t)}{N_{h}(t)} V_{h}(t)-\omega_{h} V_{h}(t)-\mu_{h} V_{h}(t) .
$$

Furthermore, the total human population is now given by $N_{h}(t)=S_{h}(t)+V_{h}(t)+E_{h}(t)+$ $I_{h}(t)+R_{h}(t)$. Using (4.1) into the basic model (2.1) gives the following vaccination model 


\begin{tabular}{|l|l|l|l|}
\hline Parameter & Description & $\begin{array}{l}\text { Baseline } \\
\text { value }\end{array}$ & Reference \\
\hline$\eta_{h}$ & $\begin{array}{l}\text { Vaccination rate of suscep- } \\
\text { tible humans }\end{array}$ & Varied & N/A \\
\hline$\omega_{h}$ & Vaccine waning rate & $\begin{array}{l}\frac{1}{5 \times 365} \\
\text { day }\end{array}$ & Estimated \\
\hline$\epsilon_{h}$ & Vaccine efficacy & $(0,1]$ & Assumed \\
\hline
\end{tabular}

Table 3: Description of vaccine-related parameters of the vaccination model (4.2).

for malaria transmission dynamics:

$$
\left\{\begin{array}{l}
\frac{d S_{h}(t)}{d t}=\Pi_{h}-b \beta_{h v} \frac{I_{v}(t)}{N_{h}(t)} S_{h}(t)-\eta_{h} S_{h}(t)+\omega_{h} V_{h}(t)-\mu_{h} S_{h}(t), \\
\frac{d V_{h}(t)}{d t}=\eta_{h} S_{h}(t)-\left(1-\epsilon_{h}\right) b \beta_{h v} \frac{I_{v}(t)}{N_{h}(t)} V_{h}(t)-\omega_{h} V_{h}(t)-\mu_{h} V_{h}(t), \\
\frac{d E_{h}(t)}{d t}=b \beta_{h v} \frac{I_{v}(t)}{N_{h}(t)}\left(S_{h}(t)+\left(1-\epsilon_{h}\right) V_{h}(t)\right)-\sigma_{h} E_{h}(t)-\mu_{h} E_{h}(t), \\
\frac{d I_{h}(t)}{d t}=\sigma_{h} E_{h}(t)-\gamma_{h} I_{h}(t)-\mu_{h} I_{h}(t)-\delta_{h} I_{h}(t), \\
\frac{d R_{h}(t)}{d t}=\gamma_{h} I_{h}(t)-\mu_{h} R_{h}(t), \\
\frac{d S_{v}(t)}{d t}=\Pi_{v}-b \beta_{v h} \frac{I_{h}(t)}{N_{h}(t)} S_{v}(t)-\mu_{v} S_{v}(t), \\
\frac{d E_{v}(t)}{d t}=b \beta_{v h} \frac{I_{h}(t)}{N_{h}(t)} S_{v}(t)-\sigma_{v} E_{v}(t)-\mu_{v} E_{v}(t) \\
\frac{d I_{v}(t)}{d t}=\sigma_{v} E_{v}(t)-\mu_{v} I_{v}(t) .
\end{array}\right.
$$

The vaccine-related parameters of the vaccination model (4.2) are described in Table 3 . It should be noted that the vaccination model (4.2) reduces to the basic model (2.1) when $\eta_{h}=\omega_{h}=0$ and $\epsilon_{h}=1$ (since this implies that $V_{h}(t) \rightarrow 0$ as $t \rightarrow \infty$ ).

\subsection{Analysis of the Vaccination Model}

The dynamics of the vaccination model (4.2) will be studied in the following feasible region: 


$$
\Omega^{*}=\Omega_{h}^{*} \cup \Omega_{v}^{*}
$$

where,

$$
\Omega_{h}^{*}=\left\{\left(S_{h}, V_{h}, E_{h}, I_{h}, R_{h}\right) \in \mathbb{R}_{+}^{5}: N_{h} \leq \frac{\Pi_{h}}{\mu_{h}}\right\}
$$

and,

$$
\Omega_{v}^{*}=\left\{\left(S_{v}, E_{v}, I_{v}\right) \in \mathbb{R}_{+}^{3}: N_{v} \leq \frac{\Pi_{v}}{\mu_{v}}\right\} .
$$

Using similar analyses as in Section 2.3, it can be shown that region $\Omega^{*}$ is positively-invariant and attracting with respect to the vaccination model (4.2). The vaccination model (4.2) has a DFE given by

$$
E_{0, v}=\left(S_{h}^{*}, V_{h}^{*}, E_{h}^{*}, I_{h}^{*}, R_{h}^{*}, S_{v}^{*}, E_{v}^{*}, I_{v}^{*}\right)=\left(S_{h}^{*}, V_{h}^{*}, 0,0,0, \frac{\Pi_{v}}{\mu_{v}}, 0,0\right),
$$

where, $S_{h}^{*}=\frac{\Pi_{h}\left(\mu_{h}+\omega_{h}\right)}{\mu_{h}\left(\mu_{h}+\omega_{h}+\eta_{h}\right)}$ and, $V_{h}^{*}=\frac{\Pi_{h} \eta_{h}}{\mu_{h}\left(\mu_{h}+\omega_{h}+\eta_{h}\right)}$. The associated next generation matrices, $F_{v}$ and $V_{v}$, for the vaccination model (4.2) are given, respectively, by

$$
F_{v}=\left[\begin{array}{cccc}
0 & 0 & 0 & \mathrm{~b} \beta_{v h} \frac{S_{h}^{*}+\left(1-\epsilon_{h}\right) V_{h}^{*}}{N_{h}^{*}} \\
0 & 0 & 0 & 0 \\
0 & \mathrm{~b} \beta_{h v} \frac{S_{v}^{*}}{N_{h}^{*}} & 0 & 0 \\
0 & 0 & 0 & 0
\end{array}\right] \text { and } V_{v}=\left[\begin{array}{cccc}
\sigma_{h}+\mu_{h} & 0 & 0 & 0 \\
-\sigma_{h} & \gamma_{h}+\mu_{h}+\delta_{h} & 0 & 0 \\
0 & 0 & \sigma_{v}+\mu_{v} & 0 \\
0 & 0 & -\sigma_{v} & \mu_{v}
\end{array}\right] \text {, }
$$

from which it follows that the vaccination reproduction number of the vaccination model (4.2), denoted by $\mathbb{R}_{v}$, is given by:

$$
\mathbb{R}_{v}=\rho\left(F_{v} V_{v}^{-1}\right)=\sqrt{\mathbb{R}_{V, h v} \mathbb{R}_{V, v h}},
$$

where,

$$
\mathbb{R}_{v, h v}=\left(\frac{b \beta_{h v} \Pi_{v} \mu_{h}}{\Pi_{h} \mu_{v}}\right)\left(\frac{\sigma_{h}}{\sigma_{h}+\mu_{h}}\right)\left(\frac{1}{\gamma_{h}+\delta_{h}+\mu_{h}}\right)
$$

and,

$$
\mathbb{R}_{v, v h}=\left(\frac{b \beta_{v h}\left(S_{h}^{*}+\left(1-\epsilon_{h}\right) V_{h}^{*}\right)}{N_{h}^{*}}\right)\left(\frac{\sigma_{v}}{\sigma_{v}+\mu_{v}}\right)\left(\frac{1}{\mu_{v}}\right) .
$$

The result below follows from Theorem 2 of [42].

Theorem 4.1 The DFE ( $\left.E_{0, v}\right)$ of the vaccination model (4.2) is locally-asymptotically stable if $\mathbb{R}_{v}<1$, and unstable if $\mathbb{R}_{v}>1$. 
Here, too, it is instructive to consider a special case of the vaccination model (4.2) without disease-induced mortality in humans (i.e., with $\delta_{h}$ set to zero) and with perfect protective vaccine efficacy (i.e., with $\epsilon_{h}=1$ ). Setting $\delta_{h}=0$ into (4.2) shows that $N_{h}(t)=\frac{\Pi_{h}}{\mu_{h}}=N_{h}^{*}$ as $t \rightarrow \infty$. It is convenient to define $\widetilde{\mathbb{R}}_{v}=\left.\mathbb{R}_{v}\right|_{\delta_{h}=0, \epsilon_{h}=1}$. We claim the following result.

Theorem 4.2 The DFE of the special case of the vaccination model (4.2), with $\delta_{h}=0$ and $\epsilon_{h}=1$, is globally-asymptotically stable in $\Omega^{*}$ whenever $\widetilde{\mathbb{R}}_{v}<1$.

Proof. Consider the vaccination model (4.2) with $\delta_{h}=0$ and $\epsilon_{h}=1$. Further, let $\widetilde{\mathbb{R}}_{0}<1$. The proof of Theorem 4.2 is based on using the comparison theorem (as in the proof of Theorem 3.2). The equations for the rate of change of the infected populations of the vaccination model (4.2) can be re-written as:

$$
\frac{d}{d t}\left(\begin{array}{c}
E_{h}(t) \\
I_{h}(t) \\
E_{v}(t) \\
I_{v}(t)
\end{array}\right)=\left(F_{v}-V_{v}\right)\left(\begin{array}{c}
E_{h}(t) \\
I_{h}(t) \\
E_{v}(t) \\
I_{v}(t)
\end{array}\right)-M_{v}\left(\begin{array}{c}
E_{h}(t) \\
I_{h}(t) \\
E_{v}(t) \\
I_{v}(t)
\end{array}\right),
$$

where the next generation matrices $F_{v}$ and $V_{v}$ are as defined in Section 4.2 and the matrix $M_{v}$ is given by:

$$
M_{v}=\left[\begin{array}{cccc}
0 & 0 & 0 & b \beta_{v h}\left(\frac{S_{h}^{*}}{N_{h}^{*}}-\frac{S_{h}}{N_{h}}\right) \\
0 & 0 & 0 & 0 \\
0 & b \beta_{h v}\left(\frac{S_{v}^{*}}{N_{h}^{*}}-\frac{S_{v}}{N_{h}}\right) & 0 & 0 \\
0 & 0 & 0 & 0
\end{array}\right] .
$$

Since $S_{h}(t) \leq S_{h}^{*}$ and $S_{v}(t) \leq S_{v}^{*}$ for all $\mathrm{t}$ in $\Omega^{*}$, it follows that the matrix $M_{v}$ is non-negative. Hence, equation (4.3) can be rewritten in terms of the following inequality:

$$
\frac{d}{d t}\left(\begin{array}{c}
E_{h}(t) \\
I_{h}(t) \\
E_{v}(t) \\
I_{v}(t)
\end{array}\right) \leq\left(F_{v}-V_{v}\right)\left(\begin{array}{c}
E_{h}(t) \\
I_{h}(t) \\
E_{v}(t) \\
I_{v}(t)
\end{array}\right)
$$

Now, consider the linear ODE system given by Equation (4.4), with the inequality replaced by equality. If $\widetilde{\mathbb{R}}_{v}<1$, then $\rho\left(F V^{-1}\right)<1$, which implies that all of the eigenvalues of $(\mathrm{F}-\mathrm{V})$ are negative. From this, it follows that the system given by Equation (4.4) is stable whenever $\widetilde{\mathbb{R}}_{v}<1$. Thus, $\left(E_{h}(t), I_{h}(t), E_{v}(t), I_{v}(t)\right) \rightarrow(0,0,0,0)$ as $t \rightarrow \infty$. Applying the standard comparison theorem [48], we see that $\left(E_{h}(t), I_{h}(t), E_{v}(t), I_{v}(t)\right) \rightarrow(0,0,0,0)$ for the nonlinear system given by the equations for $\frac{d E_{h}(t)}{d t}, \frac{d I_{h}(t)}{d t}, \frac{d E_{v}(t)}{d t}$, and $\frac{d I_{v}(t)}{d t}$ in Equation (4.2). By substituting $E_{h}=I_{h}=E_{v}=I_{v}=0$ into the remaining equations in Equation (4.2), we get $\left(S_{h}(t), V_{h}(t), E_{h}(t), I_{h}(t), R_{h}(t), S_{v}(t), E_{v}(t), I_{v}(t) \rightarrow\left(S_{h}^{*}, V_{h}^{*}, 0,0,0, S_{v}^{*}, 0,0\right)\right.$ as $t \rightarrow \infty$ when 
$\widetilde{\mathbb{R}}_{v}<1$. Thus, the DFE of the vaccination model (4.2) is globally-asymptotically stable in $\Omega^{*}$ whenever $\widetilde{\mathbb{R}}_{v}<1$. The epidemiological implication of Theorem 4.2 is that, for the special case of the vaccination model (4.2) with $\delta_{h}=0$ and $\epsilon_{h}=1$, the implementation of a routine anti-malaria immunization in the community could lead to the effective control of the disease if it results in bringing (and maintaining) the vaccination reproduction number $\left(\widetilde{\mathbb{R}}_{v}\right)$ to a value less than unity.

\subsection{Numerical Simulations}

In this section, the vaccination model (4.2) will be simulated (using the baseline values in Table 2 and Table 3, unless otherwise stated) to assess the population-level impact of vaccination in a community. In particular, the singular use of an anti-malaria vaccine and an extended IVM program (involving larviciding, adulticiding, personal protection against malaria bites, and vaccination) will be assessed.

\subsubsection{Effect of vaccination}

Vaccination is aimed at providing vaccinated susceptible individuals with some protection against the acquisition of malaria infection. In other words, vaccination is aimed at boosting the immunity of vaccinated susceptible individuals to fight against malaria infection. It is convenient to define $c_{v}=\frac{V_{h}^{*}}{N_{h}^{*}}$ to be the vaccine coverage at the disease-free steady-state. In other words, $c_{v}$ is the proportion of susceptible individuals in the community that have been vaccinated at steady-state. Figure 9 depicts a contour plot of $\mathbb{R}_{v}$, as a function of vaccine efficacy $\left(\epsilon_{h}\right)$ and coverage $\left(c_{v}\right)$, for the special case of the vaccination model (4.2) with $\delta_{h}=0$. This figure shows that the vaccination reproduction number $\left(\mathbb{R}_{v}\right)$ decreases with increasing vaccine coverage. Furthermore, even if the efficacy of the vaccine is 100\%, very high coverage of the vaccine (at least $89.2 \%$ ) will be needed to eliminate the disease in the community (i.e., to bring and maintain $\mathbb{R}_{v}<1$ ). This coverage level seems realistically unattainable. Hence, this study shows that the use of an anti-malaria vaccine alone seems may not realistically lead to malaria elimination in malaria-endemic settings. In other words, even if a highly efficacious vaccine becomes available in the near future, such vaccine has to be complemented with other anti-malaria control strategies to achieve the laudable goal of malaria elimination in a malaria-endemic community (and, subsequently, malaria eradication globally).

\subsubsection{Effect of extended integrated vector management strategy (IVM)}

In this section, the combined impact of vaccination and IVM strategy will be assessed. For these simulations, we consider two levels of the now extended IVM strategy (i.e., the previous IVM strategy implemented on the basic model (2.1) combined with a vaccination program). They are described below:

- Low Effectiveness Level of Extended IVM Strategy: here, we set larvicides at 100\% efficacy and $25 \%$ coverage (i.e., $e_{l}=1$ and $c_{l}=0.25$ ), adulticides at $50 \%$ efficacy and $25 \%$ coverage (i.e., $e_{a}=0.5$ and $c_{a}=0.25$ ), and personal protection at $50 \%$ efficacy 


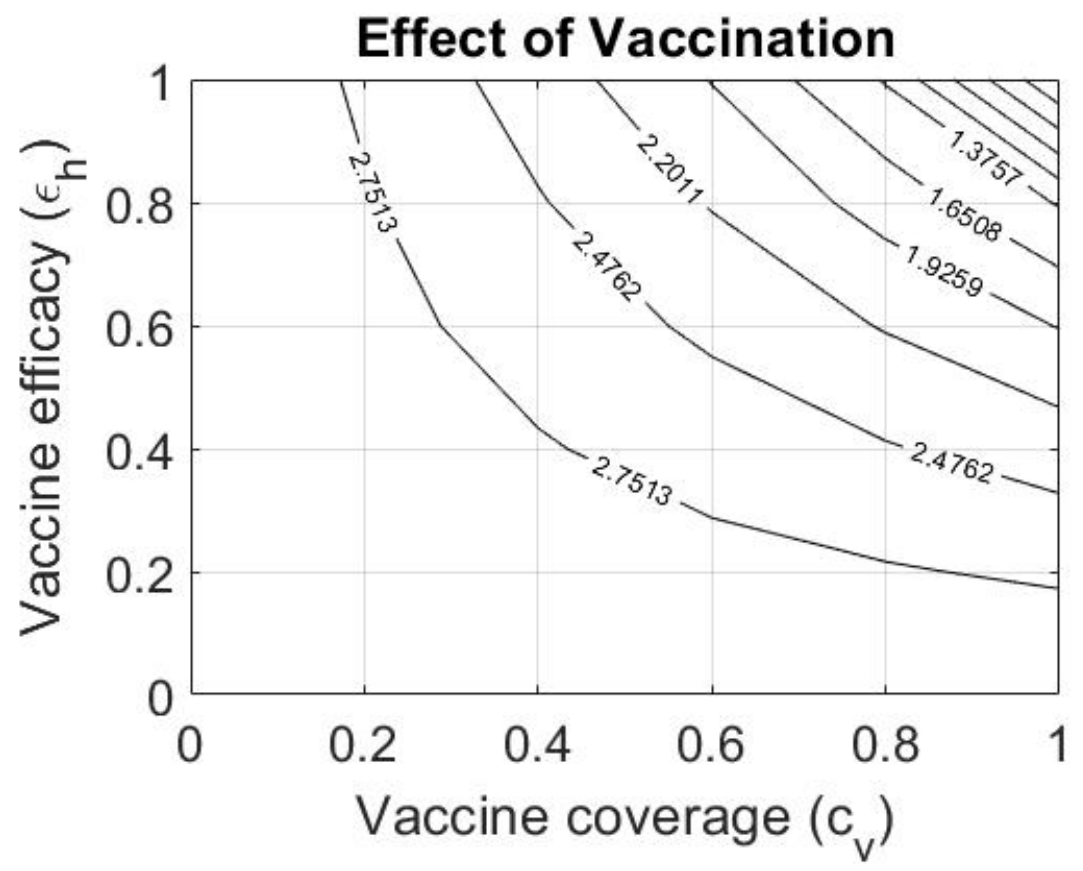

Figure 9: Contour plot of the vaccine reproduction number $\left(\mathbb{R}_{v}\right)$, for the special case of the vaccination model (4.2) with $\delta_{h}=0$, as a function of vaccine efficacy $\left(\epsilon_{h}\right)$ and coverage $\left(c_{v}\right)$ under the low effectiveness level conditions. Other parameter values are as given by the baseline values in Tables 2 and 3 , with various values of $\epsilon_{h}$ and $c_{v}$.

and $25 \%$ coverage (i.e., $e_{p}=0.5$ and $c_{p}=0.25$ ) and various vaccination effectiveness (as measured in terms of vaccine efficacy and coverage).

- Moderate Effectiveness Level of Extended IVM Strategy: In this setting, larvicides is set at $100 \%$ efficacy and $50 \%$ coverage (i.e., $e_{l}=1$ and $c_{l}=0.5$ ), adulticides at $50 \%$ efficacy and $50 \%$ coverage (i.e., $e_{a}=0.5$ and $c_{a}=0.5$ ), and personal protection at $50 \%$ efficacy and $50 \%$ coverage (i.e., $e_{p}=0.5$ and $c_{p}=0.5$ ). Various vaccination effectiveness (as measured in terms of vaccine efficacy and coverage) are considered.

As in the case of the IVM strategy formulated for the basic model (2.1), we define the reproduction number of the extended IVM strategy, denoted $\mathbb{R}_{v, m}$, as:

$$
\mathbb{R}_{v, m}=\sqrt{\mathbb{R}_{v, h v, m} \mathbb{R}_{v, v h, m}}
$$

where,

$$
\mathbb{R}_{v, h v, m}=\left(\frac{b\left(1-e_{p} c_{p} \beta_{h v} \Pi_{v}\left(1-e_{l} c_{l} \mu_{h}\right.\right.}{\Pi_{h} \mu_{v}\left(1+e_{a} c_{a}\right)}\right)\left(\frac{\sigma_{h}}{\sigma_{h}+\mu_{h}}\right)\left(\frac{1}{\gamma_{h}+\delta_{h}+\mu_{h}}\right)
$$

and,

$$
\mathbb{R}_{v, v h, m}=\left(\frac{b\left(1-e_{p} c_{p} \beta_{v h}\left(S_{h}^{*}+\left(1-\epsilon_{h}\right) V_{h}^{*}\right)\right.}{N_{h}^{*}}\right)\left(\frac{\sigma_{v}}{\sigma_{v}+\mu_{v}\left(1+e_{a} c_{a}\right)}\right)\left(\frac{1}{\mu_{v}\left(1+e_{a} c_{a}\right)}\right) .
$$




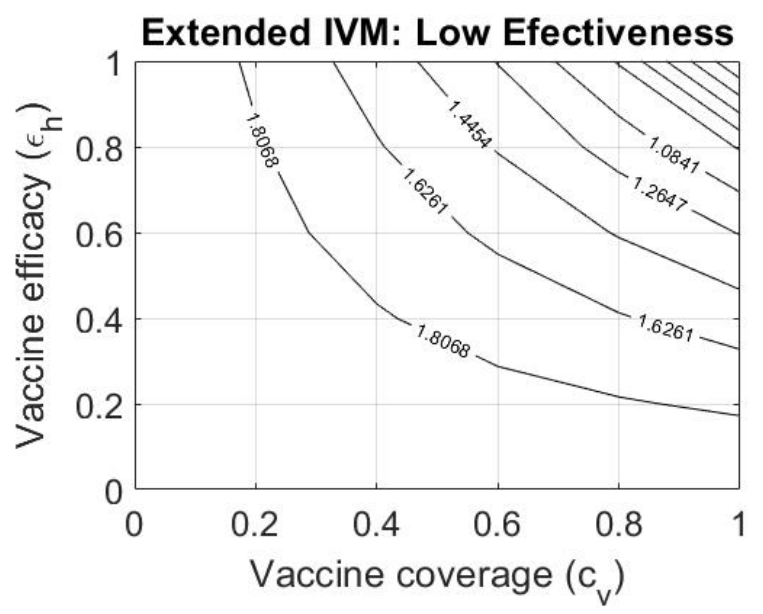

(a)

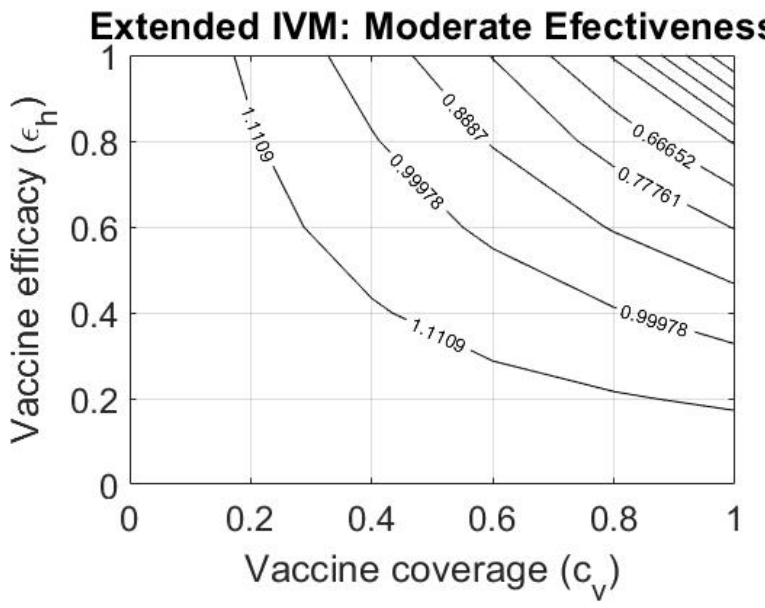

(b)

Figure 10: Simulations of the special case of the vaccination model (4.2), with $\delta_{h}=0$, for the impact of the extended IVM strategy. Contour plot of the reproduction number $\left(\mathbb{R}_{v, m}\right)$ as a function of vaccine efficacy $\left(\epsilon_{h}\right)$ and coverage $\left(c_{v}\right)$. Other parameter values are as given by the baseline values in Table 2, with various values of $e_{p}$ and $c_{p}$. (a) Low effectiveness level of extended IVM. (b) Moderate effectiveness level of extended IVM. 
Figure 10a depicts a contour plot of $\mathbb{R}_{v, m}$, as a function of vaccine efficacy $\left(\epsilon_{h}\right)$ and coverage $\left(c_{v}\right)$ under the low effectiveness level of the extended IVM strategy, for the special case of the vaccination model (4.2) with $\delta_{h}=0$. In order to apply the result of Theorem 4.2 , the plot will be analyzed at $\epsilon_{h}=1$. The figure shows that, even with $100 \%$ vaccine efficacy, a high vaccine coverage is necessary to achieve effective control of the disease in a community (i.e., bring and maintain $\mathbb{R}_{v, m}<1$ ). In particular, a vaccine coverage greater than $75.0 \%$ will be needed. This level of coverage may not be realistically attainable in malaria-endemic settings. Hence, this study suggests that the extended IVM strategy at low effectiveness level may not realistically lead to the elimination of malaria in a malaria-endemic community.

Figure $10 \mathrm{~b}$ depicts a contour plot of $\mathbb{R}_{v, m}$, as a function of vaccine efficacy $\left(\epsilon_{h}\right)$ and coverage $\left(c_{v}\right)$ under the moderate effectiveness level of the extended IVM strategy, for the special case of the vaccination model (4.2) with $\delta_{h}=0$. The figure shows that, with the $100 \%$ vaccine efficacy (needed for Theorem 4.2 to apply), malaria elimination is feasible if at least $33.9 \%$ of the unvaccinated susceptible population is vaccinated. This vaccine coverage seems reasonably attainable in malaria-endemic areas. Hence, this study shows that the prospect of malaria elimination in endemic areas using the extended IVM strategy is promising provided it is implemented at moderate effectiveness level.

\section{Discussion and Conclusion}

Malaria is a deadly infectious disease that continues to induce major public health and socio-economic burden in many parts of the world. The disease, which is spread in humans via effective bites from malaria-infected adult female Anopheles mosquitoes, accounts for millions of cases and over 500,000 deaths (mostly in children under the age of 5), on average every year. Concerted global efforts are now underway to combat or eliminate the menace of malaria. This study is based on the use of a basic mathematical model for gaining insight into the transmission dynamics of malaria in an endemic setting, and to assess the communitywide impact of various vector-based control strategies. The compartmental model, which is of the form of a deterministic system of nonlinear differential equations, was further extended to incorporate a possible vaccine for malaria.

Rigorous analysis of the basic model shows that its disease-free equilibrium is locallyasymptotically stable if the associated reproduction number is less than unity. The implication of this result is that malaria can be effectively controlled if the initial number of infected individuals or mosquitoes is small enough, provided the control measures implemented can reduce (and maintain) this reproduction number to a value less than unity. Furthermore, this equilibrium is shown to be globally-asymptotically stable (using a comparison theorem argument), for a special case of the model without malaria-induced death in the human host population, when the associated reproduction number is less than unity. For this (latter case), bringing the associated reproduction number to a value less than unity is necessary and sufficient for the effective control or elimination of malaria.

The basic model is simulated using parameter values relevant to malaria dynamics in endemic areas in sub-Saharan Africa, with focus on assessing the impact of three vectorbased control measures (namely, larviciding, adulticiding, and personal protection against mosquito bites using insect repellents) and their combinations (i.e. integrated vector man- 
agement (IVM) strategy). The simulation results obtained show that the singular implementation of any of the aforementioned strategies may not realistically lead to the elimination of malaria (although each can be effective in reducing, but not eliminating, malaria burden if their coverage and efficacy are high enough). However, combining these strategies (in an integrated vector management fashion) results in possible malaria elimination starting with the moderate effectiveness level of this (IVM) strategy. In other words, when vaccination is added to the armory of the anti-malaria control measures, the simulation results obtained in this study show that the prospects for global malaria eradication are promising if the extended IVM strategy is implemented at effectiveness level that is at least moderate.

This study can be extended in numerous directions, such as to incorporate the effect of other control strategies (e.g. gene editing strategies to modify the DNA of the mosquito), impact of anthropogenic climate change, changes in land usage, and human mobility (immigration and migration).

\section{Acknowledgments}

I am very thankful to Professor Abba Gumel (Arizona State University) for guiding and supporting me throughout this project, including during the review process. I am also very thankful to the two anonymous reviewers for their very constructive comments, which significantly enhanced the clarity of the manuscript. 


\section{References}

[1] Alphey, L., Benedict, M., Bellini, R., Clark, G. G., Dame, D. A., Service, M. W., Dobson, S. L. (2010). Sterile-Insect Methods for Control of MosquitoBorne Diseases: An Analysis. Vector-Borne and Zoonotic Diseases, 10(3), 295-311. doi:10.1089/vbz.2009.0014

[2] Anderson, R.M. and May, R.M. (1991) Infectious Disease of Humans: Dynamics and Control. Oxford University Press, Oxford.

[3] A. M. Niger and A.B. Gumel. Mathematical analysis of the role of repeated exposure on malaria transmission dynamics. Dynamical Systems and Differential Equations. 16(3)(2008): 251-287.

[4] A. Niger and A.B. Gumel. Immune response and imperfect vaccine in malaria dynamics. Mathematical Population Studies. 18(2)(2011): 54-86.

[5] Antinori S, Galimberti L, Milazzo L, Corbellino M (2012) Biology of human malaria plasmodia including Plasmodium knowlesi. Mediterr J. Hematol. Infect. Dis. 4(1):2012013

[6] Baeza, A., Santos-Vega, M., Dobson, A. P., and Pascual, M. (2017). The rise and fall of malaria under land-use change in frontier regions. Nature Ecology and Evolution, 1(5). doi:10.1038/s41559-017-0108

[7] Bhatt, S., Weiss, D., Cameron, E. et al. The effect of malaria control on Plasmodium falciparum in Africa between 2000 and 2015. Nature. 526(2015): $207 \hat{a} 211$.

[8] Bowman, C., Gumel, A., van den Driessche, P., Wu, J., Zhu, H. (2005). A mathematical model for assessing control strategies against West Nile virus. Bulletin of Mathematical Biology. 67(5)(2005): 1107â1133.

[9] Centers for Disease Control and Prevention, Anopheles Mosquitoes. (2018) Retrieved from https://www.cdc.gov/malaria/about/biology/tabs-1-5.

[10] CIA World Facebook: Ethiopia, (2017).

[11] C.N. Ngonghala, J. Mohammed, R. Zhao, O. Prosper, Interplay between insecticide treated bed-nets and mosquito demography: implications for malaria control. J. Theor. Biol. 397 (2016): 179-192.

[12] C.N. Ngonghala, S.Y.D. Valle, R. Zhao, J. Mohammed-Awel, Quantifying the impact of decay in bed-net efficacy on malaria transmission. J. Theor. Biol. 364 (2014): 247-261.

[13] Detinova, Tatiana Sergeevna, Bertram, D. S World Health Organization. (â1962)â. Age-grouping methods in diptera of medical importance, with special reference to some vectors of malaria. World Health Organization. Retrieved from https://apps.who.int/iris/handle/10665/41724. 
[14] Dong, Y., Simoes, M. L., Marois, E., Dimopoulos, G. (2018). CRISPR/Cas9-mediated gene knockout of Anopheles gambia FREP1 suppresses malaria parasite infection. PLOS Pathogens. 14(3), e1006898. doi:10.1371/journal.ppat.1006898

[15] E.A. Ashley, N.J. White, The duration of plasmodium falciparum infections. Malar. J. 13 (2014): 500 .

[16] Eikenberry, S. E. and Gumel, A. B. Mathematical modeling of climate change and malaria transmission dynamics: a historical review. Journal of Mathematical Biology. 77(4)(2018): 857-933.

[17] F. Agusto, A.B. Gumel and P.E. Parham. Qualitative assessment of the role of temperature variations on malaria transmission dynamics. Journal of Biological Systems. 23(4)(2015): 1-34.

[18] F.B. Agusto, Valle S.Y.D., Blayneh K.W., Ngonghala C.N., Goncalves M.J., Li N. The impact of bednets use on malaria prevalence. Journal of Theoretical Biology. 320(2013): $58 \hat{6} 65$.

[19] F. Demissie, A. Kebede, T. Shimels, P. Beyene, Assessment of public health implication of malaria-geohelminth co-infection with an emphasis on hookworm malaria anemia among suspected malaria patients in Asendabo, Southwest Ethiopia, Ethiop. Med. J. 47 (2) (2009): 153-158.

[20] F. Forouzannia and A.B. Gumel. Dynamics of an age-structured two-strain model for malaria transmission. Applied Mathematics and Computation. 250(2015): 860-886.

[21] From Aspiration to Action What will it take to end malaria? (2015). Retrieved from http://endmalaria2040.org/assets/Aspiration-to-Action-Dashboard.pdf.

[22] Garba, S. M., Gumel, A. B., and Abu Bakar, M. R. (2008). Backward bifurcations in dengue transmission dynamics. Mathematical Biosciences. 215(1)(2008): 11â25.

[23] Gumel, A. B. Causes of backward bifurcations in some epidemiological models. Journal of Mathematical Analysis and Applications, 395(1)(2012): 355â365.

[24] Gumel, Abba, Mccluskey, Connell and van den Driessche, P. Mathematical Study of a Staged-Progression HIV Model with Imperfect Vaccine. Bulletin of Mathematical Biology. 68(2006) 2105-2128.

[25] Iboi Enahoro, Abba Gumel and Jesse E. Taylor. Mathematical modeling of the impact of periodic release of sterile male mosquitoes and seasonality on the population abundance of malaria mosquitoes. Journal of Biological Systems. In press.

[26] IAMAT. Malaria. Retrieved from https://www.iamat.org/risks/malaria

[27] J. Mohammad-Awel and A.B. Gumel. Mathematics of an epidemiology-genetics model for assessing the role of insecticides resistance on malaria transmission dynamics. Mathematical Biosciences. 312(2019): 33-49. 
[28] J. Mohammed-Awel, Numfor E. Optimal insecticide treated bednets coverage and malaria treatment in a malaria-HIV Co-infection model. Journal of Biological Dynamics. 11(2017): 160â191.

[29] J. Mohammed-Awel, Zhao R., Numfor E., Lenhart S. Management strategies in a malaria model combining human and transmission-blocking vaccines. Discrete and Continuous Dynamical Systems - Series B (DCDS-B). 22(3)(2017): $977 \hat{a ̂ 1000 . ~}$

[30] Kamaldeen Okuneye and Abba B. Gumel. Analysis of a temperature- and rainfalldependent model for malaria transmission dynamics. Mathematical Biosciences. 287(2017): 72-92

[31] Kamaldeen Okuneye, Steffen E. Eikenberry Abba B. Gumel (2019) Weather-driven malaria transmission model with gonotrophic and sporogonic cycles, Journal of Biological Dynamics. 13(1)(2019): 288-324.

[32] Lourdes Esteva, A.B. Gumel and Cruz Vargas de Leon. Qualitative study of transmission dynamics of antibiotic-resistant malaria. Mathematical and Computer Modelling. 50(34)(2009): 611-630.

[33] Macdonald G (1952) The analysis of equilibrium in malaria. Trop. Dis. Bull. 49(9): 813 â829

[34] Macdonald G (1956) Epidemiological Basis of Malaria Control. Bull. WHO. 15:613â626

[35] Macdonald G (1956) Theory of the Eradication of Malaria. Bull. WHO. 15:369â387

[36] Macdonald G (1957) The Epidemiology and Control of Malaria. Oxford University Press, Oxford

[37] Manguin, S. et al. (2008) Biodiversity of Malaria in the World. John Libbey Eurotext

[38] M. Hailemariam, S. Gebre, Trend analysis of malaria prevalence in Arsi Negelle health center, Southwestern Ethiopia. J. Infect. Dis. Immun. 7 (1) (2015): 16.

[39] Mukhtar, A. Y. A., Munyakazi, J. B., Ouifki, R. Assessing the role of human mobility on malaria transmission. Mathematical Biosciences. (2019): 108304. doi:10.1016/j.mbs.2019.108304

[40] N. Chitnis, J.M. Hyman, J.M. Cushing, Determining important parameters in the spread of malaria through the sensitivity analysis of a mathematical model. Bull. Math. Biol. 70 (2008): 1272-1296.

[41] Ngonghala C.N., Del Valle S.Y., Zhao R., Mohammed-Awel J. Quantifying the impact of decay in bednets efficacy on malaria transmission. Journal of Theoretical Biology. 364(2014): $247 \hat{a} 261$.

[42] P. van den Driessche and James Watmough. Reproduction numbers and sub- threshold endemic equilibria for compartmental models of disease transmission. Mathematical Biosciences, 180(1)(2002): 29 â 48. 
[43] R. Carter and K.N. Mendis, Evolutionary and historical aspects of the burden of malaria. Clin. Microbiol. Rev. 15(4)(2002): 564â594. doi: 10.1128/CMR.15.4.564-594.2002.

[44] Reiner RC, Perkins TA, Barker CM, Niu T, Chaves LF, Ellis AM et al (2013) A systematic review of mathematical models of mosquito-borne pathogen transmission: 1970â2010. J. Roy. Soc. Interface. 10:20120921

[45] Smith DL, Battle KE, Hay SI, Barker CM, Scott TW, McKenzie FE (2012) Ross, Macdonald, and a theory for the dynamics and control of mosquito-transmitted pathogens. PLoS Pathog 8(4):e1002588

[46] T.S. Detinova, D.S. Bertram, World health organization, Age Grouping Methods in Diptera of Medical Importance with Special Reference to Some Vectors of Malaria, WHO Monograph Series No vol. 47, (1962).

[47] van den Berg, M., Ogutu, B., Sewankambo, N.K. et al. RTS,S malaria vaccine pilot studies: addressing the human realities in large-scale clinical trials. Trials 20(316)(2019). doi:10.1186/s13063-019-3391-7

[48] Vangipuram Lakshmikantham, Srinivasa Leela, and Anatoly A Martynyuk. Sta- bility Analysis of Nonlinear Systems. Springer, 1989.

[49] Walker, T., Moreira, L. A. (2011). Can Wolbachia be used to control malaria? Memorias Do Instituto Oswaldo Cruz, 106(suppl 1), 212â217. doi:10.1590/s007402762011000900026

[50] White M.T., Griffin J.T., Churcher T.S., Ferguson N.M., Basanez M., Ghani A.C. Modelling the impact of vector control interventions on Anopheles gambiae population dynamics. Parasites and Vectors. 4(2011): 153.

[51] World Health Organization, Malaria Fact Sheet NÂ94. (2014) Retrieved from https://www.who.int/en/news-room/fact-sheets/detail/malaria

[52] World Health Organization, World Malaria Report 2008. (2008) Retrieved from https://www.who.int/malaria/publications/atoz/9789241563697/en/.

[53] World Health Organization, World Malaria Report 2015. (2015) Retrieved from https://www.who.int/malaria/publications/world-malaria-report-2015/report/en/.

[54] World Health Organization, World Malaria Report 2019. (2019) Retrieved from https://www.who.int/publications-detail/world-malaria-report-2019.

[55] W. Sama, G. Killeen, T. Smith, Estimating the duration of Plasmodium falciparum infection from trials of indoor residual spraying. Am. J. Trop. Med. Hyg. 70 (2004): 625-634.

[56] W. Sama, S. Owusu-Agyei, I. Felger, P. Vounatsou, T. Smith. An immigration death model to estimate the duration of malaria infection when detectability of the parasite is imperfect. Stat. Med. 24(2005): 3269-3288. 
[57] Zogo B, Soma DD, Tchiekoi BN, Som $\widetilde{A}(c)$ A, Ahoua Alou LP, Koffi AA, Fournet F, Dahounto A, Coulibaly B, Kand $\tilde{A}(C)$ S, Dabir $\tilde{A}(C)$ RK, Baba-Moussa L, Moiroux N Pennetier C. 2019. Anopheles bionomics, insecticide resistance mechanisms, and malaria transmission in the Korhogo area, northern CÃŽte dâIvoire: a pre-intervention study. Parasite 26, 40. 